\title{
On Decay of Solutions and Spectral Property for a Class of Linear Parabolic Feedback Control Systems
}

\author{
Takao Nambu \\ Department of Applied Mathematics, Graduate School of System Informatics, Kobe University, Kobe, Japan \\ Email: nambu@kobe-u.ac.jp
}

Received September 4, 2013; revised October 4, 2013; accepted October 11, 2013

Copyright (C) 2013 Takao Nambu. This is an open access article distributed under the Creative Commons Attribution License, which permits unrestricted use, distribution, and reproduction in any medium, provided the original work is properly cited. In accordance of the Creative Commons Attribution License all Copyrights (C) 2013 are reserved for SCIRP and the owner of the intellectual property Takao Nambu. All Copyright (C) 2013 are guarded by law and by SCIRP as a guardian.

\begin{abstract}
Unlike regular stabilizations, we construct in the paper a specific feedback control system such that $u(t)$ decays exponentially with the designated decay rate, and that some non-trivial linear functionals of $u$ decay exactly faster than $\|u(t)\|$. The system contains a dynamic compensator with another state $v$ in the feedback loop, and consists of two states $u$ and $v$. This problem entirely differs from the one with static feedback scheme in which the system consists only of a single state $u$. To show the essential difference, some specific property of the spectral subspaces associated with our control system is studied.
\end{abstract}

Keywords: Stabilization of Linear Parabolic Systems; Decay of Functionals; Dynamic Feedback Scheme; Spectral Structures of Composite Systems; Complete Observability of Systems

\section{Introduction}

Stabilization problems for linear parabolic control systems have the history of more than three decades. Although some difficult problems are left unresolved, it seems that the study has reached a degree of maturity in a sense. The so-called dynamic compensators are introduced in the feedback loop to cope with the most difficult case such as the scheme of boundary observation/boundary input (see the literature, e.g., [1-6]). In [2-4,6], no Riesz basis is assumed, corresponding to the coefficient elliptic operators with complicated boundary operators (see (1) below). Let $H$ be a separable Hilbert space with the inner product $\langle\cdot, \cdot\rangle$ and the norm $\|\cdot\|$. A standard control system with state $u(t) \in H, t \geq 0$ consists of a finite number of inputs $g_{k}(t), 1 \leq k \leq M$, and outputs $c_{k}(u), 1 \leq k \leq N$, and is described by the following linear differential equation in $H$ :

$$
\frac{\mathrm{d} u}{\mathrm{~d} t}+L u=\sum_{k=1}^{M} g_{k}(t) h_{k}, \quad t>0, \quad u(0)=u_{0} .
$$

Here, $L$ denotes a linear closed operator with dense domain $\mathscr{T}(L)$ such that the resolvent $(\lambda-L)^{-1}$ is compact; $h_{k} \in H$ actuators through which the scalar- valued inputs $g_{k}(t)$ are inserted in the equation; and $c_{k}(u)$ linear functionals of $u$ which allow unboundedness but are subordinate to $L$. The control system also reflects boundary feedback schemes by interpreting $h_{k}$ and the differential equation in weaker topologies. In general stabilization studies, the inputs $g_{k}(t)$ are designed as a suitable feedback of the outputs $c_{k}(u)$, so that the state $u(t)$ could be stabilized as $t \rightarrow \infty$. Then every linear functional of $u$ also decays at least with the same decay rate. This is true in the case where the functional is unbounded and subordinate to $L$.

We then raise a question: can we find a nontrivial linear functional which decays faster than $\|u(t)\|$ ? The purpose of the paper is to construct a specific feedback control system such that $\|u(t)\|$ decays exponentially with the designated decay rate, and that some nontrivial linear functionals of $u(t)$, say $\langle u(t), f\rangle$, decay definitely faster than $\|u(t)\|$ for any initial state. To achieve this property, our control scheme contains a dynamic compensator with state $v(t)$ in another separable Hilbert space $\mathscr{C}$ in the feedback loop to connect $c_{k}(u(t))$ and $g_{k}(t)$. Thus the control system has state $(u(t), v(t))$ in the product space $H \times \mathscr{H}$. We note that the above decay property is achieved in a 
straightforward manner in the static feedback control scheme in which we set $M=N$ and $g_{k}(t)=c_{k}(u(t))$, $1 \leq k \leq M$. In fact, the static feedback system contains a single state $u(t)$ only, and the so-called spectral decomposition of $H$ associated with the elliptic operator enables us to find such an $f \neq 0$ in some spectral subspace. Such typical examples are the Fourier coefficients corresponding to higher frequencies. In our control system, however, it is indispensable in the spectral decomposition method to ensure a vector of the form $\boldsymbol{f}=(f, 0)$ in the spectral subspace of $H \times \mathscr{C}$ to achieve a faster decay of $\langle\boldsymbol{x}, \boldsymbol{f}\rangle_{H \times \mathscr{H}}=\langle u, f\rangle$, where $\boldsymbol{x}=(u, v)^{1}$. It is very unlikely and almost denied to find such a vector $\boldsymbol{f}=(f, 0)$ in our control system with state $(u, v)$. To make the paper clearer and more readable, unlikeliness of the above vector $\boldsymbol{f}=(f, 0)$ is discussed in detail in Section 3, which turns out to be a new spectral feature of the control system, and has never appeared in the literature; this spectral property also justifies the relevance of our problem setting.

Let us begin with the characterization of the controlled plant. Let $\Omega$ denote a bounded domain in $\mathbb{R}^{m}$ with the boundary $\Gamma$ which consists of a finite number of smooth components of $(m-1)$-dimension. Let $(\mathscr{L}, \tau)$ be a pair of linear operators defined by

$$
\begin{aligned}
& \mathscr{L} u=-\sum_{i, j=1}^{m} \frac{\partial}{\partial x_{i}}\left(a_{i j}(x) \frac{\partial u}{\partial x_{j}}\right)+\sum_{i=1}^{m} b_{i}(x) \frac{\partial u}{\partial x_{i}}+c(x) u, \\
& \tau u=\alpha(\xi) u+(1-\alpha(\xi)) \frac{\partial u}{\partial v},
\end{aligned}
$$

where $a_{i j}(x)=a_{j i}(x) \quad$ for $\quad 1 \leq i, j \leq m, \quad x \in \bar{\Omega}$; $\sum_{i, j=1}^{m} a_{i j}(x) \xi_{i} \xi_{j} \geq \delta|\xi|^{2}, \quad \forall \xi=\left(\xi_{1}, \cdots, \xi_{m}\right) \in \mathbb{R}^{m}$, $\forall x \in \bar{\Omega}$ for some positive $\delta$ and

$$
0 \leq \alpha(\xi) \leq 1, \quad \frac{\partial u}{\partial v}=\left.\sum_{i, j=1}^{m} a_{i j}(\xi) v_{i}(\xi) \frac{\partial u}{\partial x_{j}}\right|_{\Gamma},
$$

$\boldsymbol{v}(\xi)=\left(v_{1}(\xi), \cdots, v_{m}(\xi)\right)$ being the unit outer normal at $\xi \in \Gamma$. Henceforth set $H=L^{2}(\Omega)$. The pair $(\mathscr{L}, \tau)$ defines an operator $\hat{L}$ closable in $H$ as $\hat{L} u=\mathscr{L} u$ for $u \in \mathscr{V}(\hat{L})=\left\{u \in C^{2}(\Omega) \cap C^{1}(\bar{\Omega}) ; \mathscr{L} u \in L^{2}(\Omega), \tau u=0\right\}$

The closure of $\hat{L}$ is denoted as $L$. It is well known (see [7]) that $L$ has a compact resolvent $(\lambda-L)^{-1}$; that the spectrum $\sigma(L)$ lies in the complement $(\bar{\Sigma}-b)^{c}$ of some sector $\bar{\Sigma}-b$, where $\bar{\Sigma}=\left\{\lambda \in \mathbb{C} ; \theta_{0} \leq|\arg \lambda| \leq \pi\right\}$, $0<\theta_{0}<\pi / 2, b \in \mathbb{R}^{1}$; and that the following estimates hold:

${ }^{1}$ For an $\boldsymbol{f}=\left(f_{1}, f_{2}\right)$ with $f_{2} \neq 0$ in a spectral subspace of $H \times \mathscr{C}$, a faster decay of $\langle\boldsymbol{x}, \boldsymbol{f}\rangle_{H \times \%}=\left\langle u, f_{1}\right\rangle+\left\langle v, f_{2}\right\rangle_{\text {r }}$ does not ensure the same decay of $\left\langle u, f_{1}\right\rangle$.

$$
\left\|(\lambda-L)^{-1}\right\| \leq \frac{\text { const }}{1+|\lambda|}, \quad \lambda \in \bar{\Sigma}-b,
$$

where the symbol $\|\cdot\|$ also denotes the $\mathscr{L}(H)$-norm. Thus $-L$ is an infinitesimal generator of an analytic semigroup $\mathrm{e}^{-t L}, t>0$. The fractional powers $L_{c}^{\omega}$, $\omega>0$ are defined in a standard manner, where $L_{c}=L+c$ and $c>0$ is sufficiently large. It is not very clear on how the domain $\mathscr{V}\left(L_{c}^{\omega}\right)$ is characterized by the fractional Sobolev spaces, since the Dirichlet boundary is continuously connected by the Robin boundary. Such a characterization is, however, neither essential nor necessary in our study. There is a set of generalized eigenpairs $\left\{\lambda_{i}, \varphi_{i j}\right\}$ such that

$$
\begin{aligned}
& \text { 1) } \sigma(L)=\left\{\lambda_{i}\right\}_{i=1}^{\infty}, \quad \operatorname{Re} \lambda_{1} \leq \cdots \leq \operatorname{Re} \lambda_{i} \leq \cdots \rightarrow \infty \text {; } \\
& \text { 2) } \lambda_{i} \neq \lambda_{j} \text { for } i \neq j \text {; and } \\
& \text { 3) } L \varphi_{i j}=\lambda_{i} \varphi_{i j}+\sum_{k<j} \alpha_{j k}^{i} \varphi_{i k}, \quad i \geq 1, \quad 1 \leq j \leq m_{i}(<\infty) .
\end{aligned}
$$

It is well known (see, e.g., page 285 of [8]) that the set $\left\{\varphi_{i j}\right\}$ spans $H=L^{2}(\Omega)$, but does not necessarily form a Riesz basis for $H$. Let $P_{\lambda_{i}}$ be the (not necessarily orthogonal) projector corresponding to the eigenvalue $\lambda_{i}$. The restriction of $L$ onto the invariant subspace $P_{\lambda_{i}} H$ is, according to the basis $\left\{\varphi_{i j} ; 1 \leq j \leq m_{i}\right\}$, equivalent to the $m_{i} \times m_{i}$ upper triangular matrix $\Lambda_{i}$ :

$$
\left.\Lambda_{i}\right|_{(j, k)}= \begin{cases}\alpha_{k j}^{i}, & j<k, \\ \lambda_{i}, & j=k, \\ 0, & j>k .\end{cases}
$$

By setting $\Lambda_{i}=\lambda_{i}+N_{i}$, the matrix $N_{i}$ is nilpotent, that is, $N_{i}^{m_{i}}=0$. The minimum integer $n=l_{i}$ such that $\operatorname{ker} N_{i}^{n}=\operatorname{ker} N_{i}^{n+1}$ is called the ascent of $\lambda_{i}-L$. It is well known that the ascent $l_{i}$ coincides with the order of the pole $\lambda_{i}$ of $(\lambda-L)^{-1}$ (see Theorem 5.8-A of [9] for more details). Let $\left(\mathscr{L}^{*}, \tau^{*}\right)$ be the formal adjoint of $(\mathscr{L}, \tau)$ :

$$
\begin{aligned}
\mathscr{L}^{*} \varphi & =-\sum_{i, j=1}^{m} \frac{\partial}{\partial x_{i}}\left(a_{i j}(x) \frac{\partial \varphi}{\partial x_{j}}\right)-\operatorname{div}(\boldsymbol{b}(x) \varphi)+c(x) \varphi \\
\tau^{*} \varphi & =\alpha(\xi) \varphi \\
& +(1-\alpha(\xi))\left(\frac{\partial \varphi}{\partial v}+(\boldsymbol{b}(\xi) \cdot \boldsymbol{v}(\xi)) \varphi\right)
\end{aligned}
$$

where $\boldsymbol{b}(x)=\left(b_{1}(x), \cdots, b_{m}(x)\right)$. The pair $\left(\mathscr{L}^{*}, \tau^{*}\right)$ defines an operator $\hat{L}^{*}$ just as the above $\hat{L}$. Then the adjoint of $L$, denoted by $L^{*}$, is given as the closure of $\hat{L}^{*}$ in $H$. There is a set of generalized eigenpairs $\left\{\bar{\lambda}_{i}, \psi_{i j}\right\}$ such that

1) $\sigma\left(L^{*}\right)=\left\{\bar{\lambda}_{1}, \bar{\lambda}_{2}, \cdots, \bar{\lambda}_{i}, \cdots\right\}$; and

2) $L^{*} \psi_{i j}=\bar{\lambda}_{i} \psi_{i j}+\sum_{k<j} \beta_{j k}^{i} \psi_{i k}, \quad i \geq 1,1 \leq j \leq m_{i}(<\infty)$.

Similarly, the set $\left\{\psi_{i j}\right\}$ spans $H$. Setting $P_{\lambda_{i}} u=$ 
$\sum_{j=1}^{m_{i}} u_{i j} \varphi_{i j}$, we have the relationship:

$$
\begin{aligned}
& \left(\begin{array}{c}
u_{i 1} \\
\vdots \\
u_{i m_{i}}
\end{array}\right)=\Pi_{\lambda_{i}}^{-1}\left(\begin{array}{c}
\left\langle u, \psi_{i 1}\right\rangle \\
\vdots \\
\left\langle u, \psi_{i m_{i}}\right\rangle
\end{array}\right), \\
& \Pi_{\lambda_{i}}=\left(\left\langle\varphi_{i j}, \psi_{i l}\right\rangle ; \begin{array}{c}
j \rightarrow 1, \cdots, m_{i} \\
l \downarrow 1, \cdots, m_{i}
\end{array}\right) .
\end{aligned}
$$

Let us turn to the characterization of a dynamic compensator. Let $\mathscr{H}$ be any separable Hilbert space with inner product $\langle\cdot, \cdot\rangle_{\mathscr{H}}$ and norm $\|\cdot\|_{\mathscr{H}}$. Relabelling an orthonormal basis for $\mathscr{H}$, let $\left\{\eta_{i j}^{ \pm} ; i \geq 1\right.$, $\left.1 \leq j \leq n_{i}(<\infty)\right\}$ be a new orthonomal basis for $\mathscr{K}$. Every vector $v \in \mathscr{H}$ is then expressed in terms of the basis $\left\{\eta_{i j}^{ \pm}\right\}$as a Fourier series: $v=\sum_{i, j}\left(v_{i j}^{+} \eta_{i j}^{+}+v_{i j}^{-} \eta_{i j}^{-}\right)=$ $\sum_{i, j} v_{i j}^{ \pm} \eta_{i j}^{ \pm}, v_{i j}^{ \pm}=\left\langle v, \eta_{i j}^{ \pm}\right\rangle_{,}$. Let $\left\{\mu_{i}\right\}$ be a sequence of increasing positive numbers: $0<\mu_{1}<\mu_{2}<\cdots \rightarrow \infty$, and set $\omega^{ \pm}=a \pm \sqrt{-1} \sqrt{1-a^{2}}$, where $0<a<1$. Let $B$ be a linear closed operator defined as

$$
B v=\sum_{i, j} \mu_{i} \omega^{ \pm} v_{i j}^{ \pm} \eta_{i j}^{ \pm}
$$

with dense domain $\mathscr{V}(B)=\left\{v ; \sum_{i}\left|\mu_{i} v_{i j}^{ \pm}\right|^{2}<\infty\right\}$. Then, 1) $\sigma(B)=\left\{\mu_{i} \omega^{ \pm} ; i \geq 1\right\} ;$ and 2$)\left(\mu_{i} \omega^{ \pm}-B\right) \eta_{i j}^{ \pm}=0, i \geq 1$, $1 \leq j \leq n_{i}$. Thus $-B$ is the infinitesimal generator of an analytic semigroup $\mathrm{e}^{-t B}, t>0$, which is expressed by $\mathrm{e}^{-t B} v=\sum_{i, j} \mathrm{e}^{-\mu_{i} \omega^{ \pm} t} v_{i j}^{ \pm} \eta_{i j}^{ \pm}$. The semigroup $\mathrm{e}^{-t B}$ satisfies the decay estimate

$$
\left\|\mathrm{e}^{-t B}\right\|_{\mathscr{K}} \leq \mathrm{e}^{-a \mu_{1} t}, \quad t \geq 0 .
$$

The adjoint operator $B^{*}$ of $B$ is described as

$$
B^{*} v=\sum_{i, j} \mu_{i} \omega^{\mp} v_{i j}^{ \pm} \eta_{i j}^{ \pm}, v \in \mathscr{P}\left(B^{*}\right)=\mathscr{P}(B),
$$

and thus $B^{*} \eta_{i j}^{ \pm}=\mu_{i} \omega^{\mp} \eta_{i j}^{ \pm}$. Let $\mathscr{O}_{n}, n \geq 1$ be the projector in $\mathscr{H}$ such that $\mathscr{O}_{n} v=\sum_{i(\leq n), j} v_{i j}^{ \pm} \eta_{i j}^{ \pm}$.

Our control system has state $(u, v) \in H \times \mathscr{C}$, and is described as a differential equation in $H \times \mathscr{H}$ :

$$
\begin{aligned}
\frac{\mathrm{d} u}{\mathrm{~d} t}+L u & =-\left\langle v,\left(\alpha-B^{*}\right) f\right\rangle_{\mathscr{H}} \eta+\sum_{k=1}^{M}\left\langle v, \rho_{k}\right\rangle_{\mathscr{H}} \zeta_{k}, \\
\frac{\mathrm{d} v}{\mathrm{~d} t}+B v & =\sum_{k=1}^{N} c_{k}(u) \xi_{k}-\left\langle v,\left(\alpha-B^{*}\right) f\right\rangle_{\mathscr{K}} X \eta \\
& +\sum_{k=1}^{M}\left\langle v, \rho_{k}\right\rangle_{\mathscr{K}} X \zeta_{k} .
\end{aligned}
$$

The equation with state $v$ means a dynamic compensa- tor equipped with a set of outputs $\left\langle v,\left(\alpha-B^{*}\right) f\right\rangle$ and $\left\langle v, \rho_{k}\right\rangle_{\mathscr{N}}, 1 \leq k \leq M$, where $\alpha>0$. The parameters $\eta$ and $\zeta_{k}$ denote given actuators of the controlled plant, and $\xi_{k}$ actuators of the compensator to be designed. The outputs $c_{k}(u)$ of the controlled plant are considered on the boundary, and defined as

$$
c_{k}(u)= \begin{cases}\left\langle u, w_{k}\right\rangle_{\Gamma}, & \text { when } \alpha(\xi) \neq \equiv 1, \\ \left\langle\frac{\partial u}{\partial v}, w_{k}\right\rangle_{\Gamma}, & \text { when } \alpha(\xi) \equiv 1,\end{cases}
$$

where $w_{k} \in L^{2}(\Gamma)$ denotes observation weights. The operator $X \in \mathscr{L}(H ; \mathscr{K})$, specified later, denotes a unique solution to the operator equation on $\mathscr{P}(L)$,

$$
X L-B X=C, \text { where } C=-\sum_{k=1}^{N} c_{k}(\cdot) \xi_{k} .
$$

Given a suitable vector $f \in \mathscr{K}$, the control law is to construct such that the number $-\alpha$ determines the decay rate of a functional $\langle u(t, \cdot), \hat{f}\rangle$, where $\hat{f}=X^{*} f \neq 0$. As we see later, the roles of the outputs $\left\langle v(t), \rho_{k}\right\rangle$ and $\left\langle v(t),\left(\alpha-B^{*}\right) f\right\rangle$ are, respectively, to determine the decay of $u(t, \cdot)$ and the decay of $\langle u(t, \cdot), \hat{f}\rangle$. In state stabilization problems only, the output $\left\langle v(t),\left(\alpha-B^{*}\right) f\right\rangle$ does not appear. More precisely, let $\beta$ be a number such that $\alpha>\beta>0$. We seek a new feedback scheme such that the decay estimates

$$
\|u(t)\| \leq \text { conste }^{-\beta t} \text {, and }|\langle u(t), \hat{f}\rangle| \leq \text { conste }^{-\alpha t}
$$

hold for every initial value and $t \geq 0$, such that the decay of $\|u(t)\|$ is no longer improved. To achieve the non-standard decay (12), introduce a new operator

$$
L_{f}=L+\left\langle\cdot, X^{*}\left(\alpha-B^{*}\right) f\right\rangle \eta, \mathscr{V}\left(L_{f}\right)=\mathscr{V}(L),
$$

and assume conditions in terms of $L_{f}$. These conditions have never appeared in the literature. Note that conditions are posed on $L$ for state stabilization.

The functional $\langle u(t), \hat{f}\rangle$ may be regarded as a kind of output of the system. It is worthwhile to refer to our previous results on output stabilization [10-13]: In [10], the decay of outputs is discussed with lack of observability conditions, but the relationship of the decay between $u(t)$ and the outputs is unclear. In [11-13], the problem is discussed, based on a different principle, i.e., a finitedimensional pole assignment theory with constraint. The controlled plants are, however, limited to those equipped with Riesz basis, and the actuators of the controlled plant, corresponding to our $\eta$, are restrictive, and must be subject to a strong constraint: The actuators have to be designed so that their spectral elements in each spectral subspace are orthogonal to the weights of the outputs at infinity. As we have seen, the controlled plants in the present paper do not necessarily allow a Riesz basis, although a somewhat stronger condition, i.e., complete observability, is assumed. An example of systems equipped with complete observability is illustrated in the end of Section 2.

The feedback law in (9) contains parameters: $\alpha>0$, $\eta, c_{k}(\cdot), \xi_{k}, f, \rho_{k}$, and $\zeta_{k}$ to achieve the nonstandard decays (12). They are designed in the following manner: 1) The actuator $\eta \neq 0$ is given in advance arbitrarily. 2) Given the parameters $c_{k}(\cdot)$ and $\xi_{k}$, the operator solution $X$ is ensured. 3) Then, a vector $f$ is chosen among a very wide range of sets in $\mathscr{H}$. 4) Finally, $\zeta_{k}$, as well as $\rho_{k}$, are designed to satisfy a finite 
number of controllability conditions associated with the new operator $L_{f}$. It is generally desirable to pose less restrictive assumptions on the actuators of the controlled plant. In fact, $\eta \neq 0$ is arbitrary, and, as we see later that the conditions on $\zeta_{k}$ are much less restrictive than those in our preceding works above. In fact, $\zeta_{k}$ only have to be designed, belonging to an infinite dimensional subspace of $H$.

Our main results consist of Theorem 4 in Section 2 and a series of assertions in Section 3 (Theorem 7, Propositions 8, and Theorem 9): The former is on the control law ensuring the non-standard decays (12), and the latter on the relevance of the problem setting with Equation (8), which discusses a spectral property of the invariant subspaces in $H \times \mathscr{C}$ associated with the coefficient operator in (9), that is, unlikeliness of a vector of the form, $\boldsymbol{f}=\left(f_{1}, 0\right)$ in these subspaces. In Section 3, the spectrum of the coefficient operator is characterized (Theorem 9). To the best of the author's knowledge, the latter has never been discussed so far, and clarifies a new property of internal structures of control systems.

\section{Decay Estimates of Solutions}

To ensure well-posedness of our control system (8), let us begin with the operator Equation (10). Adjusting $\omega^{+}$ and $\mu_{i}, i \geq 1$, we may assume that

$$
\begin{aligned}
& \sigma(L) \cap \sigma(B)=\varnothing ; \quad \arg \omega^{+}>\theta_{0} ; \\
& \text { and } \mu_{i} \leq \mathrm{const} i^{\gamma}, i \geq 1, \text { for } 0<\exists \gamma<2
\end{aligned}
$$

(see (2) for $\theta_{0}$ ). In (9), let us express the actuators $\xi_{k}$, $1 \leq k \leq N$ as Fourier series in terms of $\left\{\eta_{i j}^{ \pm}\right\}$:

$$
\xi_{k}=\sum_{i, j}\left(\xi_{i j}^{k} \eta_{i j}^{+}+\overline{\xi_{i j}^{k}} \eta_{i j}^{-}\right), \quad \sum_{i, j}\left|\xi_{i j}^{k}\right|^{2}<\infty,
$$

where the overline denotes the complex conjugate. Before stating our first result, let us define the matrices $\Xi_{i}$, and $W_{i}, i \geq 1$ by

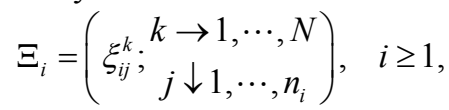

and

$$
W_{i}=\left(c_{k}\left(\varphi_{i j}\right) ; \begin{array}{l}
k \downarrow 1, \cdots, N \\
j \rightarrow 1, \cdots, m_{i}
\end{array}\right), \quad i \geq 1,
$$

respectively. Then our first result is stated as follows:

Theorem 1. 1) By assuming (14), the operator equation (11) on $(L)$ admits a unique operator solution $X \in \mathscr{L}(H ; \mathscr{H})$, which is expressed as

$$
\begin{aligned}
X u= & \sum_{k=1}^{N} \sum_{i, j}\left(c_{k}\left(\left(\mu_{i} \omega^{+}-L\right)^{-1} u\right) \xi_{i j}^{k} \eta_{i j}^{+}\right. \\
& \left.+c_{k}\left(\left(\mu_{i} \omega^{-}-L\right)^{-1} u\right) \overline{\xi_{i j}^{k}} \eta_{i j}^{-}\right) .
\end{aligned}
$$

2) Assume further that

$$
\begin{aligned}
& \operatorname{rank}\left(W_{i} \Lambda_{i}^{l} ; l \downarrow 0, \cdots, m_{i}-1\right)=m_{i}, \\
& \text { and } \operatorname{rank} \Xi_{i}=N, \quad i \geq 1 .
\end{aligned}
$$

Then we have $\overline{X^{*} \mathscr{H}}=H$. Here, $\overline{X^{*} \mathscr{H}}$ denotes the closure of $X^{*} \mathscr{H}$ in $H$.

Remark. 1) The first condition in (18), called the complete observability condition, is fulfilled with $N=1$ in the case where $\alpha_{(j+1) j}^{i} \neq 0,1 \leq j<m_{i}, i \geq 1$. Actually, by choosing the $w_{1}$ with $c_{1}\left(\varphi_{i 1}\right) \neq 0, i \geq 1$, the condition is fulfilled. In the case where $N_{i}=0, i \geq 1$, however, the condition means that $\operatorname{rank} W_{i}=m_{i}, i \geq 1$, which requires that $N$ be equal to or greater than $\sup _{i \geq 1} m_{i}$ : this is the case, for example, where $L$ is selfadjoint.

2) The condition: $\sup _{i \geq 1} m_{i}<\infty$ is the so called finite multiplicity condition. In the case of $m=1$, we know that $m_{i}=1, i \geq 1$. Thus, by choosing $N=1$, the complete observability condition is automatically fulfilled. As another example, let $L$ be a self-adjoint operator defined by $L=-\Delta$ in $\Omega=\{|x|<1\} \subset \mathbb{R}^{2}$ equipped with the Dirichlet boundary. The eigenvalues of $L$ consist of $\lambda_{m k}, \quad m \geq 0, k \geq 1$, where $\lambda_{m k}^{1 / 2}$ are the zeros of the Bessel functions $J_{m}(\cdot)$ of $m$-th order. It is expected that $\sup _{i} m_{i}=2$, if the well known Bourget's hypothesis (see pp. 484-485 of [14]) is proven. As long as the author knows, this conjecture has not been proven so far.

Proof. The result is a version of the results in [2-5], so that we give here only an outline of the proof. 1) Expression (17) and uniqueness of $X$ are examined in a straightforward manner.

2) Relation $\overline{X^{*} \mathscr{H}}=H$ is equivalent to $\operatorname{ker} X=\{0\}$. Assuming that $X u=0$, we see by (17) that

$$
\Xi_{i}\left(\begin{array}{c}
c_{1}\left(\left(\mu_{i} \omega^{+}-L\right)^{-1} u\right) \\
\vdots \\
c_{N}\left(\left(\mu_{i} \omega^{+}-L\right)^{-1} u\right)
\end{array}\right)=\mathbf{0}, \quad i \geq 1 .
$$

Since $\operatorname{rank} \Xi_{i}=N$, we see that $c_{k}\left(\left(\mu_{i} \omega^{+}-L\right)^{-1} u\right)=0$ for $i \geq 1$ and $1 \leq k \leq N$.

For a $c>0$ such that $-c \in \rho(L)$ and each $k$, we introduce a series of meromorphic functions $f_{k}^{l}(\lambda)$, $l=0,1, \cdots$ by the recursion formula:

$$
f_{k}^{0}(\lambda)=c_{k}\left((\lambda-L)^{-1} u\right), \quad f_{k}^{l+1}(\lambda)=\frac{f_{k}^{l}(\lambda)}{\lambda+c} .
$$

Each function $f_{k}^{l}(\lambda)$ has the properties: 1) It has at least the zeros $\lambda=\mu_{i} \omega^{ \pm}, i \geq 1$; and 2) the algebraic growth rate $\gamma$ of these zeros, $\mu_{i} \omega^{ \pm}$is smaller than 2 by (14). These properties combined with Carleman's theorem $[15,16]$ imply that $f_{k}^{l}(\lambda)=0$ for $\lambda \in \rho(L)$, $1 \leq k \leq N, l=0,1, \cdots$. Following [5], we calculate the residue at each $\lambda_{i}$. Then, we see that 


$$
c_{k}\left(P_{\lambda_{i}} L_{c}^{-l} u\right)=0, \quad 1 \leq k \leq N, l \geq 0 .
$$

Let $P_{\lambda_{i}} u=\sum_{1 \leq j \leq m_{i}} u_{i j} \varphi_{i j} \Leftrightarrow \boldsymbol{u}_{i}=\left(\begin{array}{lll}u_{i 1} & \cdots & u_{i m_{i}}\end{array}\right)^{\mathrm{T}}$. Note that the restriction of $L_{c}^{-l}$ on $P_{\lambda_{i}} H$ is equivalent to the matrix $\left(\Lambda_{i}+c\right)^{-l}$, and thus, $P_{\lambda_{i}} L_{c}^{-l} u \Leftrightarrow\left(\Lambda_{i}+c\right)^{-l} \boldsymbol{u}_{i}$. The relation (20) is rewritten as

$$
W_{i}\left(\Lambda_{i}+c\right)^{-l} \boldsymbol{u}_{i}=\mathbf{0}, \quad i \geq 1, l \geq 1 .
$$

The complete observability in (18) implies that $\boldsymbol{u}_{i}=\mathbf{0}$ for $i \geq 1$. In view of (5), we see that $\left\langle u, \psi_{i j}\right\rangle=0$ for every $i$ and $j$. Since the set $\left\{\psi_{i j}\right\}$ spans the whole space $H$, we conclude that $u=0$.

Q.E.D.

Decay of solutions to Equation (9): In view of (11), it is easily seen that $(X u-v)_{t}+B(X u-v)=0, t>0$, or $X u(t)-v(t)=\mathrm{e}^{-t B}\left(X u_{0}-v_{0}\right)$. Thus,

$$
\|X u(t)-v(t)\|_{\mathscr{C}} \leq \mathrm{e}^{-a \mu_{1} t}\left\|X u_{0}-v_{0}\right\|_{\mathscr{r}}
$$

for $t \geq 0$. In (9). let $\alpha<a \mu_{1}$, and $\alpha \notin \sigma(L)$ (see Proposition 2). Equation (9) contains various parameters: $\alpha, c_{k}(\cdot), \xi_{k}, \eta, f, \zeta_{k}$, and $\rho_{k}$, among which $\alpha$, $c_{k}(\cdot)$ and $\xi_{k}$ are already determined in Theorem 1. Let a non-trivial $\eta \in H$ be given arbitrarily. Then, $X \eta \neq 0$ by Theorem 1 . We find a non-trivial vector $f=$ $\sum_{i=1}^{R} \sum_{j=1}^{n_{i}}\left(f_{i j} \eta_{i j}^{+}+\overline{f_{i j}} \eta_{i j}^{-}\right) \in \mathscr{O}_{R} \mathscr{H} \quad$ such that

$$
\left\langle\xi_{k}, f\right\rangle_{\mathscr{r}}=0,1 \leq k \leq N,\langle X \eta, f\rangle_{\mathscr{r}}=1 .
$$

There is a variety of choice of such an $f$. In fact, this is simply possible, e.g., by finding $i<R$ such that

$$
\left|c_{k}\left(\left(\mu_{R} \omega^{ \pm}-L\right)^{-1} \eta\right)\right|<\left|c_{k}\left(\left(\mu_{i} \omega^{ \pm}-L\right)^{-1} \eta\right)\right|
$$

for $1 \leq k \leq N$. Then $\mathscr{R}_{R} X \eta$ does not belong to the space spanned by $\mathscr{C}_{k}, 1 \leq k \leq N$. The integer $R$ may be chosen arbitrarily large. The vectors $\zeta_{k}$ will be determined in terms of the operator $L_{f}$.

The state $u(t, \cdot)$ in (9) satisfies the equation:

$$
\begin{aligned}
& \frac{\mathrm{d}}{\mathrm{d} t} X u+B X u+C u \\
& =-\left\langle v,\left(\alpha-B^{*}\right) f\right\rangle_{\mathscr{K}} X \eta+\sum_{k=1}^{M}\left\langle v, \rho_{k}\right\rangle_{\mathscr{K}} X \zeta_{k} .
\end{aligned}
$$

Actuators $\zeta_{k}$ are chosen so that $\left\langle X \zeta_{k}, f\right\rangle_{\mathscr{N}}=0$, $1 \leq k \leq M$. An assumption on these $\zeta_{k}$ will be discussed later (see (28)). Then, it is immediately seen that

$$
\begin{aligned}
& \frac{\mathrm{d}}{\mathrm{d} t}\langle X u, f\rangle_{\mathscr{H}}+\alpha\langle X u, f\rangle_{\mathscr{H}}=\left\langle X u-v,\left(\alpha-B^{*}\right) f\right\rangle_{\mathscr{X}} \\
& =\left\langle\mathrm{e}^{-t B}\left(X u_{0}-v_{0}\right),\left(\alpha-B^{*}\right) f\right\rangle_{\mathscr{H}} .
\end{aligned}
$$

By the decay (22), we obtain the estimate:

$$
|\langle u, \hat{f}\rangle|=\left|\langle X u, f\rangle_{\mathscr{H}}\right| \leq \text { const }^{-\alpha t}
$$

for $t \geq 0$. Here, $\hat{f}=X^{*} f \in H$ is non-trivial.
The operator $L_{f}$ defined in (13) has a compact resolvent, and $\sigma\left(L_{f}\right)$ consists only of eigenvalues. Let

$$
\sigma\left(L_{f}\right)=\left\{\gamma_{i}\right\}_{i \geq 1},
$$

where $\operatorname{Re} \gamma_{1} \leq \cdots \leq \operatorname{Re} \gamma_{i} \leq \cdots \rightarrow \infty$, and $\gamma_{i} \neq \gamma_{j}$ for $i \neq j$. Each $\gamma_{i}$ may admit generalized eigenfunctions. Let $P_{i}^{f}$ be the projector corresponding to the eigenvalue $\gamma_{i}$, which is calculated as $P_{i}^{f}=\frac{1}{2 \pi \sqrt{-1}} \int_{C_{i}}\left(\lambda-L_{f}\right)^{-1} \mathrm{~d} \lambda, C_{i}$ being the small counterclockwise circle with center $\gamma_{i}$. Then,

Proposition 2. 1) The number $\alpha \notin \sigma(L)$ belongs to $\sigma\left(L_{f}\right)$. 2) Any generalized eigenfunction of $L_{f}$ in $P_{i}^{f} H \quad\left(\gamma_{i} \neq \alpha\right)$ and $X^{*} f$ are orthogonal to each other.

Proof. 1) Suppose that there is a $\chi \neq 0$ such that $\left(\alpha-L_{f}\right) \chi=0$. Then, $(\alpha-L) \chi=\left\langle\chi, X^{*}\left(\alpha-B^{*}\right) f\right\rangle \eta$. Thus we have, as a necessary condition,

$$
\chi=\left\langle\chi, X^{*}\left(\alpha-B^{*}\right) f\right\rangle(\alpha-L)^{-1} \eta
$$

Now set $\chi=(\alpha-L)^{-1} \eta(\neq 0)$, and calculate as

$$
\begin{aligned}
\left\langle\chi, X^{*}\left(\alpha-B^{*}\right) f\right\rangle & =\left\langle X \chi,\left(\alpha-B^{*}\right) f\right\rangle_{\mathscr{X}} \\
& =\alpha\langle X \chi, f\rangle_{\mathscr{H}}-\langle X L \chi, f\rangle_{\mathscr{K}} \\
& =\langle X(\alpha-L) \chi, f\rangle_{\mathscr{X}} \\
& =\langle X \eta, f\rangle_{\mathscr{X}}=1 .
\end{aligned}
$$

Thus we see that

$$
\alpha \chi=L \chi+\eta=L \chi+\left\langle\chi, X^{*}\left(\alpha-B^{*}\right) f\right\rangle \eta=L_{f} \chi .
$$

2) Let $\gamma \in \sigma\left(L_{f}\right), \gamma \neq \alpha$, and $W_{\gamma}^{(k)}=\left\{\chi ;\left(\gamma-L_{f}\right)^{k} \chi=0\right\}, k \geq 1$, possible generalized eigenspaces of $\gamma$. For a $\chi \in W_{\gamma}^{(1)}$, we calculate by (23) as

$$
\begin{aligned}
\gamma\left\langle\chi, X^{*} f\right\rangle & =\left\langle L \chi, X^{*} f\right\rangle+\left\langle\chi, X^{*}\left(\alpha-B^{*}\right) f\right\rangle \\
& =\langle(X L-B X) \chi, f\rangle_{\mathscr{X}}+\alpha\left\langle\chi, X^{*} f\right\rangle \\
& =\langle C \chi, f\rangle_{\mathscr{X}}+\alpha\left\langle\chi, X^{*} f\right\rangle=\alpha\left\langle\chi, X^{*} f\right\rangle
\end{aligned}
$$

This implies that $\left\langle\chi, X^{*} f\right\rangle=0$, or $W_{\gamma}^{(1)} \perp X^{*} f$. Suppose then that $W_{\gamma}^{(k)} \perp X^{*} f, i \geq 1$. For a $\chi \in W_{\gamma}^{(k+1)}$, the function $\left(\gamma-L_{f}\right) \chi$ is in $W_{\gamma}^{(k)}$, and $\left\langle\left(\gamma-L_{f}\right) \chi, X^{*} f\right\rangle=0$. The same calculation as above immediately shows that $\left\langle\chi, X^{*} f\right\rangle=0$. Thus,

$$
X^{*} f \perp \bigcup_{k \geq 1} W_{\gamma}^{(k)} .
$$

The integer $k$ varies over a finite set of positive integers depending on $\gamma$.

Q.E.D.

We now choose the actuators $\zeta_{k}$ in (9) such that

$$
\zeta_{k} \in \overline{\bigcup_{\gamma_{i} \neq \alpha}\left(\bigcup_{i \geq 1} W_{\gamma_{i}}^{(k)}\right)}, \quad 1 \leq k \leq M
$$


Then, $\zeta_{k} \perp X^{*} f$ by the above proposition. All parameters except for $\rho_{k}$ in (9) are determined.

Rewrite the equation for $u(t)$ in (9) as

$$
\begin{aligned}
& \frac{\mathrm{d} u}{\mathrm{~d} t}+L_{f} u-\sum_{k=1}^{M}\left\langle u, X^{*} \rho_{k}\right\rangle \zeta_{k} \\
& =\left\langle X u-v,\left(\alpha-B^{*}\right) f\right\rangle_{\mathscr{H}} \eta-\sum_{k=1}^{M}\left\langle X u-v, \rho_{k}\right\rangle_{\mathscr{H}} \zeta_{k} .
\end{aligned}
$$

Let us introduce an operator $L_{f f}$ as

$$
L_{f f}=L+\left\langle\cdot, X^{*}\left(\alpha-B^{*}\right) f\right\rangle \eta-\sum_{k=1}^{M}\left\langle\cdot, c_{k}\right\rangle \zeta_{k} .
$$

Let the integer $l$ be such that $l=\min \left\{i ; \alpha<\operatorname{Re} \gamma_{i}\right\}$. Then, $\alpha=\gamma_{l-1}, l \geq 2$. The following proposition is just a simple version of the result in [17].

Proposition 3. Let $\hat{P}$ be the projector defined by $\hat{P}=\sum_{\substack{1 \leq i \leq l, 1 \\ i \neq l}}^{f}$. Choose a $\beta$ such that $0<\beta<\alpha$. Suppose that the pair $\left(\left.L_{f}\right|_{\hat{P} H},\left\{\hat{P} \zeta_{1}, \cdots, \hat{P} \zeta_{M}\right\}\right)$ is a controllable one. Then we find $c_{k} \in \hat{P}^{*} H$ such that

$$
\min \operatorname{Re} \sigma\left(L_{f f}\right)=\beta ; \quad \beta \in \sigma\left(L_{f f}\right) .
$$

Thus, $\left\|\mathrm{e}^{-t L_{f f}}\right\| \leq C \mathrm{e}^{-\beta t}, t \geq 0$. We are ready to state the non-standard deay of solutions to Equation (9).

Theorem 4. Let $0<\beta<\alpha<a \mu_{1}$ such that $\alpha \notin \sigma(L)$. Suppose that

1) $\xi_{k}$ and $c_{k}(\cdot)$ satisfy the rank conditions (18);

2) $\eta \neq 0$ is arbitrarily given;

3) $f \in \mathscr{O}_{R} \quad$ is chosen to satisfy (23); and

4) $\zeta_{k} \in \overline{\bigcup_{\gamma_{i} \neq \alpha}\left(\bigcup_{k \geq 1} W_{\gamma_{i}}^{(k)}\right)}$ satisfiy the controllability condition in Proposition 3.

Then we find a large integer $n$; vectors $\rho_{k} \in$ Pि $_{n}$; and a postive $\tilde{\beta}<\alpha$ close to $\beta$, and subsequently the control system in the product space $H \times{ }_{n} \mathscr{H}$;

$$
\begin{aligned}
& \frac{\mathrm{d} u}{\mathrm{~d} t}+L u=-\left\langle v_{1},\left(\alpha-B^{*}\right) f\right\rangle_{\text {, }_{n}{ }^{\prime}} \eta \\
& +\sum_{k=1}^{M}\left\langle v_{1}, \rho_{k}\right\rangle_{\text {夰 }} \zeta_{k} \text {, } \\
& \frac{\mathrm{d} v_{1}}{\mathrm{~d} t}+B_{1} v=\sum_{k=1}^{N} c_{k}(u) \cdot \mathscr{P}_{n} \xi_{k}+\sum_{k=1}^{M}\left\langle v_{1}, \rho_{k}\right\rangle_{n_{n} \mathscr{N}} X \zeta_{k} \\
& -\left\langle v_{1},\left(\alpha-B^{*}\right) f\right\rangle_{n} X \eta,
\end{aligned}
$$

where $B_{1}=\left.B\right|_{\text {. Every solution }}\left(u(t, \cdot), v_{1}(t)\right) \in$ $H \times P_{n} \mathscr{H}$ satisfies the decay estimate:

$$
\begin{aligned}
& \|u(t, \cdot)\|+\left\|v_{1}(t)\right\|_{\mathscr{H}} \leq \text { conste }^{-\tilde{\beta} t}\left(\left\|u_{0}\right\|+\left\|v_{10}\right\|_{\mathscr{C}}\right), \\
& |\langle u(t, \cdot), \hat{f}\rangle| \leq \text { conste }^{-\alpha t}\left(\left\|u_{0}\right\|+\left\|v_{10}\right\|_{\mathscr{H}}\right)
\end{aligned}
$$

for $t \geq 0$. The estimates for $\|u(t, \cdot)\|$ and $\left\|v_{1}(t)\right\|_{\mathscr{H}}$ can be no longer improved.

Proof. Choose the functions $c_{k} \in \hat{P}^{*} H, 1 \leq k \leq M$ stated in Proposition 3. In view of Theorem 1, we find $\rho_{k} \in \mathscr{H}$ such that $X^{*} \rho_{k}$ arbitrarily approximate $c_{k}$ in the topology of $H$. Since $\mathscr{H}$ is separable, we may assume with no loss of generality that these $\rho_{k}$ are constructed in $\mathscr{P}_{n} \mathscr{H}$ for an enough large $n>R$.

Let us consider the operator $L_{f f}$ and the perturbed $\tilde{L}_{f f}=L_{f}-\sum_{k=1}^{M}\left\langle\cdot, X^{*} \rho_{k}\right\rangle \zeta_{k}$. The right-hand side of (29) is dominated by the decay estimate (22). Since $X^{*} \rho_{k}$ are chosen close to $c_{k}$, the semigroup $\mathrm{e}^{-t \tilde{L}_{f f}}$ is stable, and satisfies the standard estimate:

$$
\left\|\mathrm{e}^{-t \tilde{L}_{f f}}\right\| \leq C \mathrm{e}^{-(\beta-C \delta) t}, \quad t \geq 0,
$$

where $\delta=\sum_{k}\left\|X^{*} \rho_{k}-c_{k}\right\|\left\|\zeta_{k}\right\|$. Thus every solution $(u(t, \cdot), v(t))$ to Equation (9) satisfies the estimate:

$$
\|u(t, \cdot)\|+\|v(t)\|_{\mathscr{C}} \leq \text { conste }^{-\hat{\beta} t}\left(\|u(0)\|+\left\|v_{0}\right\|\right),
$$

where $\hat{\beta}=\beta-C \delta>0$. The decay estimate for the functional $\langle u(t, \cdot), \hat{f}\rangle$ is already obtained in (25).

The control system (30) is derived in the following manner: Set $v_{1}(t)=V_{n} v(t)$, and apply the projector to the equation for $v$ in (9). By noting that $\left(\alpha-B^{*}\right) f \in \mathscr{P}_{R} \mathscr{H}$, then, (30) is immediately obtained. Equation (30) is clearly well posed in $H \times \mathscr{P}_{n} \mathscr{O C}$ : Thus every solution to (30) is derived from the solution $(u(t, \cdot), v(t))$ to (9) with initial value $\left(u_{0}, v_{0}\right)$ such that $\mathscr{O}_{n} v_{0}=v_{10}$, by setting $v_{1}(t)=\mathscr{P}_{n} v(t)$. Thus the first estimate of (31) is derived from (33). The second estimate of (31) is clear by (25).

Finally we show that the first estimate of (31) for $\left(u(t, \cdot), v_{1}(t)\right)$ is no more improved. The spectrum $\sigma\left(\tilde{L}_{f f}\right)$ of the perturbed operator $\tilde{L}_{f f}$ consists only of eigenvalues. It is expected that the value of $\min \operatorname{Re} \sigma\left(\tilde{L}_{f f}\right)$ would be close to $\beta=\min \operatorname{Re} \sigma\left(L_{f f}\right)$ as long as $X^{*} \rho_{k}$ are close to $c_{k}$ : When both $L_{f f}$ and $\tilde{L}_{f f}$ are selfadjoint, it is well known-via the min-max principle (see [18]) - that each eigenvalue of $L_{f f}$ is continuous relative to the coefficient parameters. In our problem, the following result holds:

Proposition 5. The minimum of $\operatorname{Re} \sigma\left(\tilde{L}_{f f}\right)$ is continuous relative to $\left\|X^{*} \rho_{k}-c_{k}\right\|, \quad 1 \leq k \leq M$.

Proof. Set $\tilde{L}_{f f}=L_{f f}+\sum_{\mathrm{l} \leq k \leq M}\left\langle\cdot, c_{k}-X^{*} \rho_{k}\right\rangle \zeta_{k}=L_{f f}+D_{\varepsilon}$. In view of (32), the left half-plane: $\operatorname{Re} \lambda<\beta-C \delta$ is contained in $\rho\left(\tilde{L}_{f f}\right)$. Thus we see that $\beta-C \delta \leq$ $\min \operatorname{Re} \sigma\left(\tilde{L}_{f f}\right)$. Choose an $r>0$ enough small so that $\{\lambda ;|\lambda-\beta|<r\} \cap \sigma\left(L_{f f}\right)=\{\beta\}$. Let $\Gamma$ be the counterclockwise circle: $\Gamma=\{\lambda ;|\lambda-\beta|=r\}$, and suppose that $\left\|\left(\lambda-L_{f f}\right)^{-1}\right\| \leq d=d(r)$ for $\lambda \in \Gamma$. Choose $X^{*} \rho_{k}$ such that $\left\|D_{\varepsilon}\right\| \leq \delta<(2 d)^{-1}$. Then, $\Gamma$ belongs to $\rho\left(\tilde{L}_{f f}\right)$. In fact, we have the relation:

$$
\begin{aligned}
& \left(\lambda-\tilde{L}_{f f}\right)^{-1}=\left(\lambda-L_{f f}\right)^{-1}\left(1-D_{\varepsilon}\left(\lambda-L_{f f}\right)^{-1}\right)^{-1}, \\
& \left\|\left(\lambda-\tilde{L}_{f f}\right)^{-1}\right\| \leq 2 d, \quad \lambda \in \Gamma .
\end{aligned}
$$

Recall that, for $\lambda \in \rho\left(L_{f f}\right) \cap \rho\left(\tilde{L}_{f f}\right)$, the (second) resolvent equation: 


$$
\left(\lambda-L_{f f}\right)^{-1}-\left(\lambda-\tilde{L}_{f f}\right)^{-1}=-\left(\lambda-L_{f f}\right)^{-1} D_{\varepsilon}\left(\lambda-\tilde{L}_{f f}\right)^{-1}
$$

holds. Then we see that

$$
\begin{aligned}
& \frac{1}{2 \pi i} \int_{\Gamma}\left(\lambda-L_{f f}\right)^{-1} u \mathrm{~d} \lambda-\frac{1}{2 \pi i} \int_{\Gamma}\left(\lambda-\tilde{L}_{f f}\right)^{-1} u \mathrm{~d} \lambda \\
& =\frac{-1}{2 \pi i} \int_{\Gamma}\left(\lambda-L_{f f}\right)^{-1} D_{\varepsilon}\left(\lambda-\tilde{L}_{f f}\right)^{-1} u \mathrm{~d} \lambda, \quad \forall u \in H .
\end{aligned}
$$

The first term of the above left-hand side of (35) is the projector, corresponding to the eigenvalue $\beta$ of $L_{f f}$. Choose $X^{*} \rho_{k}$ closer to $c_{k}$, if necessary, so that

$$
\delta=\sum_{k=1}^{M}\left\|X^{*} \rho_{k}-c_{k}\right\|\left\|\zeta_{k}\right\|<\min \left(\frac{1}{2 d}, \frac{1}{2 d^{2} r}, r\right) .
$$

Supposing that $\sigma\left(\tilde{L}_{f f}\right)$ is contained in the half-plane: $\{\lambda ; \operatorname{Re} \lambda>\beta+r\}$, we then derive a contradiction. If so, the resolvent $\left(\lambda-\tilde{L}_{f f}\right)^{-1} u$ is analytic inside and on $\Gamma$. Thus the second term of the left-hand side of (35) must be equal to 0 . Let $u$ be an eigenfunction of $L_{f f}$, corresponding to the eigenvalue $\beta$. Then,

$$
u=\frac{-1}{2 \pi i} \int_{\Gamma}\left(\lambda-L_{f f}\right)^{-1} D_{\varepsilon}\left(\lambda-\tilde{L}_{f f}\right)^{-1} u \mathrm{~d} \lambda \neq 0 .
$$

The right-hand side is, however, estimated as follows:

$$
\begin{aligned}
& \left\|\frac{-1}{2 \pi i} \int_{\Gamma}\left(\lambda-L_{f f}\right)^{-1} D_{\varepsilon}\left(\lambda-\tilde{L}_{f f}\right)^{-1} u \mathrm{~d} \lambda\right\| \\
& \leq \frac{1}{2 \pi} \int_{\Gamma}\left\|\left(\lambda-L_{f f}\right)^{-1} D_{\varepsilon}\left(\lambda-\tilde{L}_{f f}\right)^{-1} u\right\||\mathrm{d} \lambda| \\
& \leq 2 d^{2} r\left\|D_{\varepsilon}\right\|\|u\|<\|u\|,
\end{aligned}
$$

which is a contradiction. Therefore, the spectrum $\sigma\left(\tilde{L}_{f f}\right)$ also lies in the left-half plane: $\{\lambda \in \mathbb{C} ; \operatorname{Re} \lambda \leq \beta+r\}$. As a conclusion, the minimum of $\operatorname{Re} \sigma\left(\tilde{L}_{f f}\right)$ satisfies the estimate:

$$
\beta-C r \leq \min \operatorname{Re} \sigma(\bar{M}) \leq \beta+r,
$$

as long as $\left\|X^{*} \rho_{k}-c_{k}\right\|, 1 \leq k \leq M$ are small. Q.E.D.

Let us turn to the proof of Theorem 4. Choose an $r$ in Proposition 5 such that $\beta+r<\alpha$. Let $v_{1}$ be the eigenvalue of $\tilde{L}_{f f}$ such that $\operatorname{Re} v_{1}=\min \operatorname{Re} \sigma\left(\tilde{L}_{f f}\right)$, and $u_{0}$ a corresponding eigenfunction:

$$
\left(v_{1}-\tilde{L}_{f f}\right) u_{0}=0, \quad \operatorname{Re} v_{1} \leq \beta+r<\alpha .
$$

Set $v_{0}=X u_{0}$. As easily seen from Equation (29), the function $(u(t, \cdot), v(t))$ given by

$$
u(t, \cdot)=\mathrm{e}^{-v_{1} t} u_{0}, \quad v(t)=X u(t, \cdot)=\mathrm{e}^{-v_{1} t} v_{0}
$$

is the solution to Equation (9) with the initial value $\left(u_{0}, v_{0}\right)$. In view of the reduction process to Equation (30), the function $\left(\mathrm{e}^{-v_{1} t} u_{0}, \mathrm{e}^{-v_{1} t} \cdot{ }_{n} / v_{0}\right)$ is thus a non-trivial solution to (30). This shows that the decay (31) for $\|u(t, \cdot)\|+\left\|v_{1}(t)\right\|_{\mathscr{R}}$ is no longer improved. This finishes the proof of Theorem 4 .

Q.E.D.
Example. In (1), let us consider the case where $\Omega$ is a bounded interval $I=(0,1) \subset \mathbb{R}^{1}$. The pair of differential operators $(\mathscr{L}, \tau)$ is then rewritten as

$$
\begin{gathered}
\mathscr{L} u=-\left(a(x) u_{x}\right)_{x}+b(x) u_{x}+c(x) u, \\
\tau u=\alpha(\xi) u+(1-\alpha(\xi)) u_{v}, \quad \xi=0,1,
\end{gathered}
$$

where $\alpha(0), \alpha(1) \in[0,1]$, and $a(x) \geq \delta(>0)$. Let $T \in \mathscr{L}(H)$ be an operator defined, for $u \in H$, by

$$
T u=\kappa(x) u, \quad \kappa(x)=\exp \left(-\int_{0}^{x} \frac{b(s)}{2 a(s)} \mathrm{d} s\right) .
$$

Clearly $T$ defines an isomorphism in $H$. Let us consider the case where $\tau$ is of the third kind, i.e., $\alpha(0)$, $\alpha(1)<1$. Then, $T$ transforms $(\mathscr{L}, \tau)$ into another pair $(\tilde{L}, \tilde{\tau})$, which defines a self-adjoint operator $\tilde{L}$ with dense domain $\mathscr{C}(\tilde{L})$. In $(\tilde{L}, \tilde{\tau}), a$ is unchanged; $b$ and $c$ are changed, respectively, to 0 and $c-(\kappa b)^{\prime} /(2 \kappa)$; and $\tilde{\tau}$ of the third kind. The idea is a slightly modified version of the well known result (see page 292 of [18]). Based on this, we have

Proposition 6. 1) The spectrum $\sigma(L)$ consists of real and simple eigenvalues: $\sigma(L)=\left\{\lambda_{i}\right\}_{i=1}^{\infty}, \quad \lambda_{1}<\lambda_{2}<$ $\cdots<\lambda_{i}<\cdots \rightarrow \infty$.

2) The eigenfunctions $\varphi_{i}(x)$ of $\lambda_{i}$ forms a Riesz basis. Any $u \in H$ is uniquely expressed as $u=\sum_{i=1}^{\infty} c_{i} \varphi_{i}$.

In our problem, we know that $m_{i}=1, i \geq 1$. Thus we choose $N=1$, so that the output of the system is a single observation at the end point $x=0$ as

$$
u(t, 0)=\langle u(t, \cdot), w\rangle_{\Gamma},
$$

that is, $w(0)=1, w(1)=0$. Let us examine some assumptions in Theorem 4 in this example. Most important is the complete observability (18). The matrices $W_{i}$ in (16) are now $W_{i}=c_{1}\left(\varphi_{i}\right)=\varphi_{i}(0) \neq 0, i \geq 1$. Thus, we see that the complete observability is satisfied. Since $L_{f}$ in (13) is a one dimensional operator, the multiplicities of the eigenvalues $\gamma_{i}$ are equal to 1 . This enables us to choose $M=1$ in (9). In Proposition 3, the controllability condition on the actuator $\zeta_{1} \in \overline{\bigcup_{\gamma_{i} \neq \alpha}\left(W_{\gamma_{i}}^{(1)}\right)}$ is stated as follows: let $\chi_{i}$ be eigenfunctions of $\gamma_{i}$. By setting $\hat{P} \zeta_{1}=\sum_{i \neq l-1}^{1 \leq i \leq l} c_{i} \chi_{i}$, the controllability condition is simply that $c_{i} \neq 0, \quad 1 \leq i \leq l, i \neq l-1$.

In the case where $\tau$ is of the first kind, the output is a single observation at the end point $x=0$ as $u_{x}(t, 0)=\left\langle u_{x}(t, \cdot), w\right\rangle$. Proposition 6 also holds in this case. Since $W_{i}=c_{1}\left(\varphi_{i}\right)=-\varphi_{i}^{\prime}(0) \neq 0, i \geq 1$, the complete observability is similarly satisfied.

\section{Spectral Property of the Coefficient Operator}

We go back to the problem raised in Section 1: Unlikeliness of a vector of the form $\left(\begin{array}{ll}f_{1} & 0\end{array}\right)^{\mathrm{T}}$. The basic 
control system is Equation (30) in the product space $H \times \mathscr{O}_{n} \mathscr{H}$. To avoid any unnecessary technical complexity, we limit ourselves to the simple case of one dimensioanl equations raised in (36), $\tau$ being of the third kind. In the setting of the space $\mathscr{C}$ as well as $B$ in (6), we can choose $n_{i}=1, i \geq 1$. Thus, $M=N=1$. Equation (30) is simply rewritten as

$$
\frac{\mathrm{d}}{\mathrm{d} t}\left(\begin{array}{l}
u \\
v
\end{array}\right)+A\left(\begin{array}{l}
u \\
v
\end{array}\right)=0,\left.\left(\begin{array}{l}
u \\
v
\end{array}\right)\right|_{t=0} \in H \times \mathscr{O}_{n} \mathscr{C},
$$

where $A$ is defined as

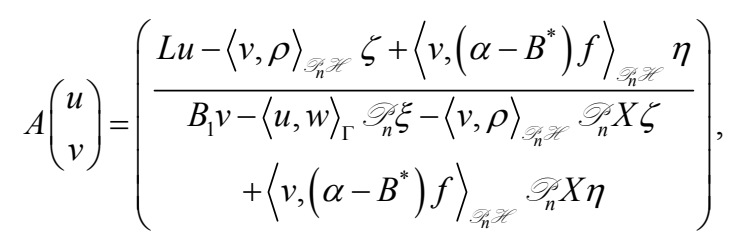

and $\mathscr{P}(A)=\mathscr{P}(L) \times \mathscr{P}_{n} \mathscr{H}$. Here, $\langle u, w\rangle_{\Gamma}=u(0)$ (see (37)). The operator $A$ is sectorial, and every solution to (30) or (38) is expressed as $(u(t) v(t))^{\mathrm{T}}=\mathrm{e}^{-t A}\left(u_{0} v_{10}\right)^{\mathrm{T}}$, $t \geq 0$. Let $\boldsymbol{P}$ be the projector corresponding to a $\lambda \in \sigma(A)$ with $\operatorname{Re} \lambda \geq \alpha$. In view of the relation:

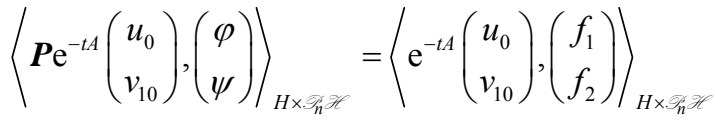

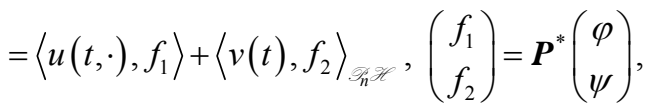

the right-hand side of which decays as $t \rightarrow \infty$ with decay rate $-\alpha$ for every initial state. Now we ask: Does the range of the $\boldsymbol{P}^{*}$ contain a vector of the form $\left(\begin{array}{ll}f_{1} & 0\end{array}\right)^{\mathrm{T}}$ ? This problem immediately leads to the structure of the eigenspaces of the operator $A^{*}$. In the operator $A$, the vectors $\rho \in \mathscr{P}_{n} \mathscr{H}$ and $f \in \mathscr{R}_{R} \mathscr{C}$ of the compensator are the parameters to be designed $(n>R)$. In designing these parameters, they are generally influenced by small perturbations. It is thus implausible to assume that some Fourier coefficients of these parameters would be designed to be $0:$ such conditions are very easily broken. Thus we may henceforth assume that

$$
\begin{aligned}
& \rho_{i}^{ \pm} \neq 0, \quad 1 \leq i \leq n, \\
& \tilde{f}_{i}^{ \pm}=\left(\alpha-\mu_{i} \omega^{\mp}\right) f_{i}^{ \pm} \neq 0, \quad 1 \leq i \leq R,
\end{aligned}
$$

where $\rho_{i}^{ \pm}$and $f_{i}^{ \pm}$denote, respectively, $\left\langle\rho, \eta_{i}^{ \pm}\right\rangle_{\text {, }}$ and $\left\langle f, \eta_{i}^{ \pm}\right\rangle_{\text {的 }}$. The actuators $\zeta$ and $\eta$ of the controlled plant are the given parameters in advance. It is also implausible to assume that some Fourier coefficients of $\zeta$ and $\eta$ relative to $L^{*}$ might be equal to 0 . Thus we may also assume that

$$
\left\langle\zeta, \psi_{i}\right\rangle \neq 0, \quad\left\langle\eta, \psi_{i}\right\rangle \neq 0, \quad i \geq 1 .
$$

The main results in this section are Theorem 7, Proposition
8, and Theorem 9 stated just below. The proof of these results will be given later.

Theorem 7. Let $1 \leq K \leq \min (R, n-R+1)$. Suppose that $\left\{\alpha_{k}\right\}_{k=1}^{K} \subset \sigma\left(A^{*}\right)$ and that

$$
A^{*}\left(\begin{array}{c}
p_{k} \\
q_{k}
\end{array}\right)=\alpha_{k}\left(\begin{array}{c}
p_{k} \\
q_{k}
\end{array}\right), \quad\left(\begin{array}{c}
p_{k} \\
q_{k}
\end{array}\right) \neq\left(\begin{array}{l}
0 \\
0
\end{array}\right), \quad 1 \leq k \leq K,
$$

where $\alpha_{i} \neq \alpha_{j}$ for $i \neq j$. Then any linear combination of these eigenvectors $\left(p_{k} q_{k}\right)^{\mathrm{T}}$ of $A^{*}$ cannot generate $a$ vector of the form, $\left(f_{1} 0\right)^{\mathrm{T}}, f_{1} \neq 0$.

Remark. The adjoint operator $A^{*}$ will be characterized later in (49). Theorem 7 also asserts that there is no eigenvector of the form, $\left(\begin{array}{ll}f_{1} & 0\end{array}\right)^{\mathrm{T}}, f_{1} \neq 0$. The restriction on $K$ is derived from our setting of the operator $A$ in (39): The setting is made for constructing a finitedimensional compensator. In the original Equation (9), however, the parameters are constructed in a more general setting. The operator $A$ is then replaced by

$$
\begin{aligned}
& A\left(\begin{array}{l}
u \\
v
\end{array}\right)=\left(\begin{array}{c}
\frac{L u-\langle v, \rho\rangle_{\mathscr{C}} \zeta+\left\langle v,\left(\alpha-B^{*}\right) f\right\rangle_{\not{C}} \eta}{B v-\langle u, w\rangle_{\Gamma} \xi-\langle v, \rho\rangle_{\overparen{ }} X \zeta} \\
+\left\langle v,\left(\alpha-B^{*}\right) f\right\rangle_{\not ூ} X \eta
\end{array}\right), \\
& \mathscr{P}(A)=\mathscr{P}(L) \times \mathscr{P}(B) \text {. }
\end{aligned}
$$

Then, the above restriction on the $K$ is removed: In fact, the integer $K$ may be chosen arbitrarily large.

We hope to know more on $\sigma\left(A^{*}\right)$. The following proposition partly gives concrete informations on what $\sigma\left(A^{*}\right)$ consists of. It shows that $\sigma\left(B_{1}\right)$ is contained in $\sigma\left(A^{*}\right)$, regardless of the assumptions (40) and (41).

Proposition 8. The numbers $\mu_{i} \omega^{ \pm}, 1 \leq i \leq n$ belong to $\sigma\left(A^{*}\right)$. Actually we have the relations:

$$
A^{*}\left(\begin{array}{c}
-X^{*} \eta_{i}^{\mp} \\
\eta_{i}^{\mp}
\end{array}\right)=\mu_{i} \omega^{ \pm}\left(\begin{array}{c}
-X^{*} \eta_{i}^{\mp} \\
\eta_{i}^{\mp}
\end{array}\right)
$$

for $1 \leq i \leq n$. Since the set $\left\{\eta_{i}^{\mp}\right\}$ forms an orthonormal system for $\mathscr{C}$, any linear combinations of these eigenvectors cannot generate a vector of the form, $\left(\begin{array}{ll}f_{1} & 0\end{array}\right)^{\mathrm{T}}, \quad f_{1} \neq 0$.

To seek eigenvalues of $A^{*}$ other than $\left\{\mu_{i} \omega^{ \pm}\right\}_{i=1}^{n}$, let us recall the operator $\tilde{L}_{f f}$ which appeared in $(32)$, where $\tilde{L}_{f f}=L-\left\langle\cdot, X^{*} \rho\right\rangle \zeta+\left\langle\cdot X^{*}\left(\alpha-B^{*}\right) f\right\rangle \eta$ with $\mathscr{P}\left(\tilde{L}_{f f}\right)=$ $\mathcal{O}(L)$. The adjoint operator $\tilde{L}_{f f}^{*}$ is clearly given by $\tilde{L}_{f f}^{*}=$ $L^{*}-\langle\cdot \zeta\rangle X^{*} \rho+\langle\cdot, \eta\rangle X^{*}\left(\alpha-B^{*}\right) f$ with $\mathscr{Q}\left(\tilde{L}_{f f}^{*}\right)=\mathscr{O}\left(L^{*}\right)$. In the following result, we characterize $\sigma\left(A^{*}\right)$ by introducing an operator $L_{g}^{*}$, a slightly perturbed operator of $\tilde{L}_{f f}^{*}$ :

Theorem 9. Let $L_{g}^{*}$ be an operator defined as

$$
\begin{aligned}
& L_{g}^{*}=L^{*}+\langle\cdot, \eta\rangle X_{1}^{*}\left(\alpha-B^{*}\right) f-\langle\cdot, \zeta\rangle X_{1}^{*} \rho, \\
& \mathscr{P}\left(L_{g}^{*}\right)=\mathscr{Y}\left(L^{*}\right),
\end{aligned}
$$

where $X_{1}=\mathscr{R}_{n} X$. Then, we have the relation 


$$
\sigma\left(A^{*}\right)=\sigma\left(L_{g}^{*}\right) \cup \sigma\left(B_{1}^{*}\right) .
$$

Let $\{\hat{\lambda}, \hat{\psi}\}$ be an arbitrary eigenpair of $L_{g}^{*}$ such that $\hat{\lambda}$ is not contained in $\sigma\left(B_{1}^{*}\right)$. Then, the corresponding eigenvector $\left(\begin{array}{ll}p & q\end{array}\right)^{\mathrm{T}}$ of $A^{*}$ is given by

$$
\left(\begin{array}{c}
\hat{\psi}-X_{1}^{*}\left(\hat{\lambda}-B_{1}^{*}\right)^{-1}\left(-\langle\hat{\psi}, \zeta\rangle \rho+\langle\hat{\psi}, \eta\rangle\left(\alpha-B^{*}\right) f\right) \\
\left(\hat{\lambda}-B_{1}^{*}\right)^{-1}\left(-\langle\hat{\psi}, \zeta\rangle \rho+\langle\hat{\psi}, \eta\rangle\left(\alpha-B^{*}\right) f\right)
\end{array}\right)
$$

where $\left(\hat{\lambda}-B_{1}^{*}\right)^{-1}\left(-\langle\hat{\psi}, \zeta\rangle \rho+\langle\hat{\psi}, \eta\rangle\left(\alpha-B^{*}\right) f\right) \neq 0$.

In the above assertions, we need to characterize the adjoint operator $A^{*}$, which will be described later by (51). To seek the structure of $A^{*}$, let us begin with the operator equation:

$$
X_{1} L-B_{1} X_{1}=C_{1}, C_{1}=-\langle\cdot, w\rangle_{\Gamma} \xi_{1}, \xi_{1}=\mathscr{O}_{n} \xi .
$$

It is clear that (47) admits a unique solution $X_{1} \in \mathscr{L}\left(H ; \mathscr{P}_{n}\right)$, and that the solution is expressed as $X_{1}=\mathscr{P}_{n} X$ (see (17)). Let $g \in H^{2}(I)$ be a unique solution to the boundary value problem: $\left(\mathscr{L}^{*}+c\right) g=0$ in $I, \tau^{*} g=w$ on $\Gamma$. We note that $\|g\|$ remains bounded when $c \rightarrow \infty$ (this fact will be used in Lemma 10 below). For any $\lambda \in \rho(L)$, note that

$$
\begin{aligned}
\left.(\lambda-L)^{-1} u\right|_{x=0} & =\left\langle(\lambda-L)^{-1} u, w\right\rangle_{\Gamma} \\
& =\left\langle u, L_{c}^{*}\left(\bar{\lambda}-L^{*}\right)^{-1} g\right\rangle, L_{c}^{*}=L^{*}+c .
\end{aligned}
$$

Then the adjoint $X_{1}^{*} \in \mathscr{L}\left(\mathscr{O}_{n} \mathscr{C} ; H\right)$ is expressed as

$$
\begin{aligned}
X_{1}^{*} v= & \sum_{i \leq n}\left(v_{i}^{+} \bar{\xi}_{i} L_{c}^{*}\left(\mu_{i} \omega^{-}-L^{*}\right)^{-1} g+v_{i}^{-} \xi_{i} L_{c}^{*}\left(\mu_{i} \omega^{+}-L^{*}\right)^{-1} g\right) \\
= & -\left\langle v, \xi_{1}\right\rangle_{\text {, }{ }_{n}} g+\sum_{i \leq n} v_{i}^{+} \bar{\xi}_{i}\left(\mu_{i} \omega^{-}+c\right)\left(\mu_{i} \omega^{-}-L^{*}\right)^{-1} g \\
& +\sum_{i \leq n} v_{i}^{-} \xi_{i}\left(\mu_{i} \omega^{+}+c\right)\left(\mu_{i} \omega^{+}-L^{*}\right)^{-1} g,
\end{aligned}
$$

where $v=\sum_{i \leq n} v_{i}^{ \pm} \eta_{i}^{ \pm}, v_{i}^{ \pm}=\left\langle v, \eta_{i}^{ \pm}\right\rangle_{\text {, sol }}$. Thus,

$$
X_{1}^{*} v \in H^{2}(I), \tau^{*}\left(X_{1}^{*} v\right)=-\left\langle v, \xi_{1}\right\rangle_{\text {, 夰 }} w .
$$

Let us find the equation for $X_{1}^{*}$. For $u \in \mathscr{V}(L)$ and $v \in \mathscr{P}_{n} \mathscr{H}$, we calculate through Green's formula, (47), and the boundary condition (48) as

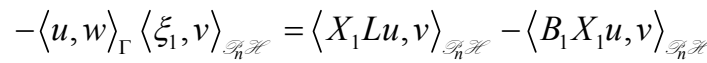

$$
\begin{aligned}
& =\left\langle L u, X_{1}^{*} v\right\rangle-\left\langle X_{1} u, B_{1}^{*} v\right\rangle_{\text {层花 }} \\
& =\left\langle u, \mathscr{L}^{*} X_{1}^{*} v\right\rangle-\left\langle\xi_{1}, v\right\rangle_{\text {织 }}\langle u, w\rangle_{\Gamma} \\
& -\left\langle u, X_{1}^{*} B_{1}^{*} v\right\rangle \text {, }
\end{aligned}
$$

and thus $\left\langle u, \mathscr{L}^{*} X_{1}^{*} v\right\rangle-\left\langle u, X_{1}^{*} B_{1}^{*} v\right\rangle=0$. Since $\mathscr{V}(L)$ is dense in $H$, we see that

$$
\mathscr{L}^{*} X_{1}^{*} v=X_{1}^{*} B_{1}^{*} v, \quad \forall v \in \mathscr{P}_{n} \mathscr{H} .
$$

Let us calculate the adjoint $A^{*}$. By assuming that $p$ is in $H^{2}(I)$ and satisfies the boundary condition: $\tau^{*} p=$

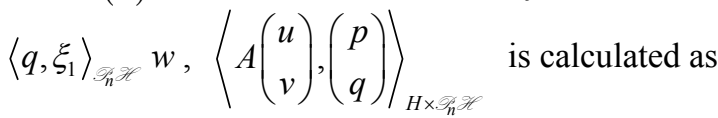

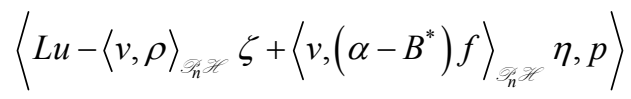

$$
\begin{aligned}
& +\left\langle B_{1} v-\langle u, w\rangle_{\Gamma} \xi_{1}-\langle v, \rho\rangle_{\text {, }{ }_{n}} X_{1} \zeta\right. \\
& \left.+\left\langle v,\left(\alpha-B^{*}\right) f\right\rangle_{\text {, }} X_{1} \eta, q\right\rangle_{\text {, }} \\
& =\left\langle u, \mathscr{L}^{*} p\right\rangle+\left\langle u, \tau^{*} p\right\rangle_{\Gamma}-\langle v, \rho\rangle_{\text {, }_{n} /}\langle\zeta, p\rangle \\
& +\left\langle v,\left(\alpha-B^{*}\right) f\right\rangle_{\text {, }{ }_{n} \mathscr{K}}\langle\eta, p\rangle \\
& +\left\langle v, B_{1}^{*} q\right\rangle_{\text {, }}-\langle u, w\rangle_{\Gamma}\left\langle\xi_{1}, q\right\rangle_{\text {, }} \\
& -\langle v, \rho\rangle_{\text {, }}\left\langle X_{1} \zeta, q\right\rangle_{\text {. }} \\
& +\left\langle v,\left(\alpha-B^{*}\right) f\right\rangle_{\text {, }}\left\langle X_{1} \eta, q\right\rangle_{\text {, }} \\
& =\left\langle u, \mathscr{L}^{*} p\right\rangle+\left\langle v, B_{1}^{*} q-\left\langle p+X_{1}^{*} q, \zeta\right\rangle \rho\right. \\
& \left.+\left\langle p+X_{1}^{*} q, \eta\right\rangle\left(\alpha-B^{*}\right) f\right\rangle_{\text {, }{ }_{n \pi}} \\
& =\left\langle\left(\begin{array}{l}
u \\
v
\end{array}\right), A^{\dagger}\left(\begin{array}{l}
p \\
q
\end{array}\right)\right\rangle_{H \times \text {, 当 }},
\end{aligned}
$$

where $A^{\dagger}\left(\begin{array}{l}p \\ q\end{array}\right)$ is given by

$$
\left(\begin{array}{c}
\mathscr{L}^{*} p \\
B_{1}^{*} q-\left\langle p+X_{1}^{*} q, \zeta\right\rangle \rho+\left\langle p+X_{1}^{*} q, \eta\right\rangle\left(\alpha-B^{*}\right) f
\end{array}\right),
$$

and $\mathscr{Y}\left(A^{\dagger}\right)=\left\{\left(\begin{array}{ll}p & q\end{array}\right)^{T} ; p \in H^{2}(I), q \in \mathscr{O P}_{n}, \tau^{*} p-\right.$ $\left.\left\langle q, \xi_{1}\right\rangle_{\text {, }} w=0\right\}$. We see that $\mathscr{Y}\left(A^{\dagger}\right) \subset \mathscr{V}\left(A^{*}\right)$, and thus $A^{\dagger} \subset A^{*}$. In order to show that $A^{\dagger}=A^{*}$, we need the following elementary result:

Lemma 10. The operator $A^{\dagger}$ is densely defined, and the bounded inverse, $A_{c}^{\dagger-1}=\left(A^{\dagger}+c\right)^{-1}$ exists for a sufficiently large $c>0$.

Proof. Given a $\left(\begin{array}{ll}u & v\end{array}\right)^{\mathrm{T}} \in H \times \operatorname{OP}_{n}$, we solve the equation: $A_{c}^{\dagger}\left(\begin{array}{ll}p & q\end{array}\right)^{\mathrm{T}}=\left(\begin{array}{ll}u & v\end{array}\right)^{\mathrm{T}}$, where $A_{\mathrm{c}}^{\dagger}=A^{\dagger}+c$. Set $\tilde{f}=\left(\alpha-B^{*}\right) f$, and define an operator $\hat{B}^{\mathrm{C}}$ as

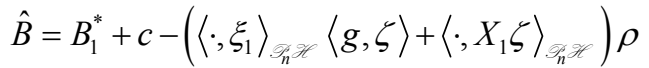

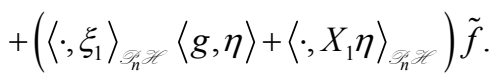

The function $g$ depends on $c$. However, since $g$ remains bounded as $c \rightarrow \infty$, there is a bounded inverse: $\hat{B}^{-1} \in \mathscr{L}\left(\mathscr{O}_{n}\right)$ for a sufficiently large $c>0$. A straightforward calculation shows that $\left(\begin{array}{ll}p & q\end{array}\right)^{\mathrm{T}}$ defined by $p=\left\langle q, \xi_{1}\right\rangle_{\text {, } \mathscr{K}} g+L_{c}^{*-1} u$ and $q=\hat{B}^{-1}\left(v+\left\langle L_{c}^{*-1} u, \zeta\right\rangle \rho\right.$ 
$\left.-\left\langle L_{c}^{*-1} u, \eta\right\rangle \tilde{f}\right)$ uniquely solves the above equation. Thus the bounded inverse $A_{c}^{\dagger}$ exists.

Denseness of $\mathscr{P}\left(A^{\dagger}\right)$ : it is enough to show that

$$
\left\langle A_{c}^{\dagger-1}\left(\begin{array}{l}
u \\
v
\end{array}\right),\left(\begin{array}{l}
\varphi \\
\psi
\end{array}\right)\right\rangle_{H \times, \bar{n} \not \mathcal{C}}=0 \quad \text { for } \forall\left(\begin{array}{l}
u \\
v
\end{array}\right)
$$

implies $\left(\begin{array}{ll}\varphi & \psi\end{array}\right)^{\mathrm{T}}=\left(\begin{array}{ll}0 & 0\end{array}\right)$. The above left-hand side is calculated for every $(u v)^{\mathrm{T}} \in H \times \mathscr{P}_{n} \mathscr{P C}$ as

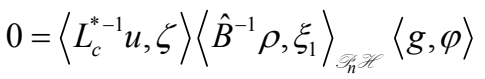

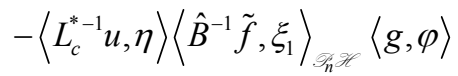

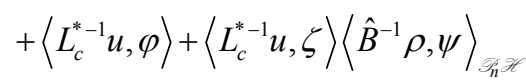

$$
\begin{aligned}
& -\left\langle L_{c}^{*-1} u, \eta\right\rangle\left\langle\hat{B}^{-1} \tilde{f}, \psi\right\rangle_{\text {. }}
\end{aligned}
$$

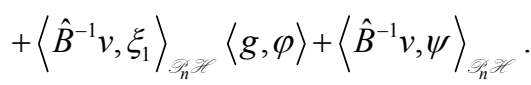

Since $\mathscr{P}\left(L_{c}^{*}\right)$ is dense, this means that

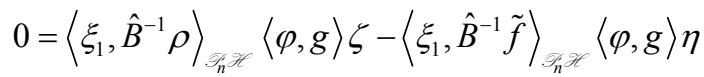

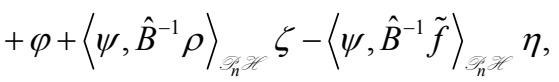

and $0=\langle\varphi, g\rangle \xi_{1}+\psi$, from which we conclude that $\varphi=0$ and $\psi=0$.

Q.E.D.

In view of the fact that both $A_{c}^{*-1}=\left(A_{c}^{-1}\right)^{*}$ and $A_{c}^{\dagger-1}$ exist as a bounded inverse, it is immediate that $\mathscr{P}\left(A^{c}\right)$ is contained in $\left(A^{\dagger}\right)$. We have proven that

$$
A^{*}=A^{\dagger} \text {. }
$$

Proof of Proposition 8. By setting $p=-X_{1}^{*} q$ and $q=\eta_{i}^{\mp}, \quad 1 \leq i \leq n, \quad\left(\begin{array}{ll}p & q\end{array}\right)^{\mathrm{T}}$ belongs to $\mathscr{P}\left(A^{*}\right)$. By (49), we see that $B_{1}^{*} q=\mu_{i} \omega^{ \pm} q$, and $\mathscr{L}^{*} p=-X_{1}^{*} B_{1}^{*} q=$ $-\mu_{i} \omega^{ \pm} X_{1}^{*} q=\mu_{i} \omega^{ \pm} p$, which shows (43) Q.E.D.

Proof of Theorem 7. Assuming that $\sum_{k=1}^{K} q_{k}=0$ in (42), we derive a contradiction. In (42), adding the equations of $q_{k}$ over 1 through $K$, we see that

$$
-c_{1} \rho+c_{2} \tilde{f}=\sum_{k=1}^{K} \alpha_{k} q_{k}=\sum_{k=1}^{K-1}\left(\alpha_{k}-\alpha_{K}\right) q_{k},
$$

where $\quad c_{1}=\sum_{k=1}^{K}\left\langle p_{k}, \zeta\right\rangle, \quad c_{2}=\sum_{k=1}^{K}\left\langle p_{k}, \eta\right\rangle, \quad$ and $\tilde{f}=\left(\alpha-B^{*}\right) f$. The Fourier coefficients of these vectors relative to the orthonormal system $\left\{\eta_{i}^{ \pm}\right\}$satisfy

$$
-c_{1} \rho_{i}^{ \pm}+c_{2} \tilde{f}_{i}^{ \pm}=\sum_{k=1}^{K-1}\left(\alpha_{k}-\alpha_{K}\right) q_{k i}^{ \pm}, \quad i \geq 1,
$$

where $q_{k i}^{ \pm}=\left\langle q_{k}, \eta_{i}^{ \pm}\right\rangle$. Note that $\tilde{f}_{i}^{ \pm}=\left(\alpha-\mu_{i} \omega^{\mp}\right) f_{i}^{ \pm}=$ 0 for $i>R$. We show that $c_{1}=0$. Supposing the contrary, we must have

$$
\rho_{i}^{ \pm}=-\sum_{k=1}^{K-1} \frac{\alpha_{k}-\alpha_{K}}{c_{1}} q_{k i}^{ \pm}, \quad R<i \leq n .
$$

Set $d_{k}=\left\langle p_{k}+X_{1}^{*} q_{k}, \zeta\right\rangle, \quad 1 \leq k \leq K$ for simplicity.
Then, $\sum_{k=1}^{K} d_{k}=c_{1}$. In the equation for $q_{k}, 1 \leq k \leq$ $K-1$, we see that

$$
\mu_{i} \omega^{\mp} q_{k i}^{ \pm}+d_{k} \sum_{j=1}^{K-1} \frac{\alpha_{j}-\alpha_{K}}{c_{1}} q_{j i}^{ \pm}=\alpha_{k} q_{k i}^{ \pm}, R<i \leq n .
$$

The number of these $\mu_{i} \omega^{\mp}$ is $2(n-R)$. Consider the algebraic equation in $\tau$ :

$$
\left|\begin{array}{cccc}
\tau+d_{1} \frac{\alpha_{1}-\alpha_{K}}{c_{1}}-\alpha_{1} & d_{1} \frac{\alpha_{2}-\alpha_{K}}{c_{1}} & \cdots & d_{1} \frac{\alpha_{K-1}-\alpha_{K}}{c_{1}} \\
d_{2} \frac{\alpha_{1}-\alpha_{K}}{c_{1}} & \tau+d_{2} \frac{\alpha_{2}-\alpha_{K}}{c_{1}}-\alpha_{2} & \cdots & d_{2} \frac{\alpha_{K-1}-\alpha_{K}}{c_{1}} \\
\vdots & \vdots & \ddots & \vdots \\
d_{K-1} \frac{\alpha_{1}-\alpha_{K}}{c_{1}} & d_{K-1} \frac{\alpha_{2}-\alpha_{K}}{c_{1}} & \cdots & \tau+d_{K-1} \frac{\alpha_{K-1}-\alpha_{K}}{c_{1}}-\alpha_{K-1}
\end{array}\right|=0 .
$$

The equation admits $(K-1)$ solutions. The number of the solutions $\tau$ which agree with one of the $\mu_{i} \omega^{\mp}$ is at most $(K-1)$. In other words, the determinant is not equal to 0 for the other $\mu_{i} \omega^{\mp}$, the number of which is atleast $2(n-R)-(K-1)>0$. Thus for these $\mu_{i} \omega^{\mp}$, we must have $q_{1 i}^{ \pm}=q_{2 i}^{ \pm}=\cdots=q_{(K-1) i}^{ \pm}=0$. By (53), this implies that $\rho_{i}^{ \pm}=0$, which contradicts our assumption (40). We have shown that $c_{1}=0$. Thus we have, for $i>1$,

$$
c_{2} \tilde{f}_{i}^{ \pm}=\sum_{k=1}^{K-1}\left(\alpha_{k}-\alpha_{K}\right) q_{k i}^{ \pm} .
$$

Comparing the Fourier coefficients in the equations to $q_{k}$ in (42), we see that

$$
\begin{aligned}
& \mu_{i} \omega^{\mp} q_{k i}^{ \pm}-d_{k} \rho_{i}^{ \pm}=\alpha_{k} q_{k i}^{ \pm}, \text {or } \\
& \left(\mu_{i} \omega^{\mp}-\alpha_{k}\right) q_{k i}^{ \pm}=d_{k} \rho_{i}^{ \pm}, R<i \leq n, 1 \leq k \leq K-1 .
\end{aligned}
$$

The number of the eigenvalues $\alpha_{k}$ which agree with one of the $\mu_{i} \omega^{\mp}$ is at most $(K-1)$. In other words, the number of the $\mu_{i} \omega^{\mp}$ which does not agree with any of the $\alpha_{k}$ is at least $2(n-R)-(K-1)>0$. For these $\mu_{i} \omega^{\mp} \neq \alpha_{k}$, we see from (55) that

$$
0=\sum_{k=1}^{K-1}\left(\alpha_{k}-\alpha_{K}\right) q_{k i}^{ \pm}=\sum_{k=1}^{K-1} \frac{d_{k}\left(\alpha_{k}-\alpha_{K}\right)}{\mu_{i} \omega^{\mp}-\alpha_{k}} \rho_{i}^{ \pm} .
$$

Since $\rho_{i}^{ \pm} \neq 0$, this means that the relation in $\sigma$ :

$$
0=\sum_{k=1}^{K-1} \frac{d_{k}\left(\alpha_{k}-\alpha_{K}\right)}{\sigma-\alpha_{k}}
$$

holds for the above $\sigma=\mu_{i} \omega^{\mp}$, the distinct number of which is at least $2(n-R)-(K-1) \geq K-1$. This implies that the relation (56) holds for any $\sigma(\in \mathbb{C}) \neq \alpha_{1}, \cdots, \alpha_{K-1}$. Calculating the residue at each $\alpha_{k}$, we find that $d_{1}=d_{2}=\cdots=d_{K-1}=0$, and thus $d_{K}=c_{1}-\sum_{k=1}^{K-1} d_{k}=0$, too.

We go back to the equations to $q_{k}$ in (42) again. Since $d_{k}=0,1 \leq k \leq K$, we have

$$
B_{1}^{*} q_{k}+\left\langle p_{k}+X_{1}^{*} q_{k}, \eta\right\rangle \tilde{f}=\alpha_{k} q_{k}, \quad 1 \leq k \leq K .
$$


Set $e_{k}=\left\langle p_{k}+X_{1}^{*} q_{k}, \eta\right\rangle$. Then, $\sum_{k=1}^{K} e_{k}=c_{2}$. Calculating the Fourier coefficients, we have

$$
\left(\mu_{i} \omega^{\mp}-\alpha_{k}\right) q_{k i}^{ \pm}=-e_{k} \tilde{f}_{i}^{ \pm}, \quad 1 \leq i \leq R, 1 \leq k \leq K .
$$

The numbers of these $\mu_{i} \omega^{\mp}$ and $\alpha_{k}$ are $2 R$ and $K-1$, respectively. Thus, the number of $\mu_{i} \omega^{\mp}$ which does not agree with any of $\alpha_{k}$ is at least $2 R-K \geq K$. For these $\mu_{i} \omega^{\mp} \neq \alpha_{k}$, we see by the relation (54) that

$$
\begin{aligned}
c_{2} \tilde{f}_{i}^{ \pm} & =\sum_{k=1}^{K-1}\left(\alpha_{k}-\alpha_{K}\right) q_{k i}^{ \pm} \\
& =-\sum_{k=1}^{K-1} \frac{e_{k}\left(\alpha_{k}-\alpha_{K}\right)}{\mu_{i} \omega^{\mp}-\alpha_{k}} \tilde{f}_{i}^{ \pm} .
\end{aligned}
$$

But, since $\tilde{f}_{i}^{ \pm} \neq 0$ by (40), this means that the relation in $\sigma$ :

$$
\begin{aligned}
& c_{2}=-\sum_{k=1}^{K-1} \frac{e_{k}\left(\alpha_{k}-\alpha_{K}\right)}{\sigma-\alpha_{k}}, \\
& \text { or } \quad c_{2} \prod_{k=1}^{K-1}\left(\sigma-\alpha_{k}\right) \\
& \quad+\sum_{k=1}^{K-1} e_{k}\left(\alpha_{k}-\alpha_{K}\right) \prod_{j=1, j \neq k}^{K-1}\left(\sigma-\alpha_{j}\right)=0
\end{aligned}
$$

holds for the above $\sigma=\mu_{i} \omega^{\mp}$, the distinct number of which is at least $2 R-(K-1) \geq K-1$. The situation is the same as in (56). Thus the relation (57) holds for any $\sigma \neq \alpha_{1}, \cdots, \alpha_{K-1}$. Calculating the residue at each $\alpha_{k}$, we similarly find that $e_{1}=\cdots=e_{K-1}=0, c_{2}=0$, and thus $e_{K}=c_{2}-\sum_{k=1}^{K-1} e_{k}=0$, too. Since $d_{k}=e_{k}=0,1 \leq k \leq K$, we have finally obtained from (42) and (55) that $B_{1}^{*} q_{k}=$ $\alpha_{k} q_{k}, \quad 1 \leq k \leq K$, and $\sum_{k=1}^{K-1}\left(\alpha_{k}-\alpha_{K}\right) q_{k}=c_{2} \tilde{f}=0$. Applying $B_{1}^{* l}$ to the both sides of the above second equation, we see that, for $l=1,2, \cdots$,

$$
B_{1}^{* l} \sum_{k=1}^{K-1}\left(\alpha_{k}-\alpha_{K}\right) q_{k}=\sum_{k=1}^{K-1}\left(\alpha_{k}-\alpha_{K}\right) \alpha_{k}^{l} q_{k}=0 .
$$

In other words, we have the relation:

$$
\left(\begin{array}{cccc}
1 & 1 & \cdots & 1 \\
\alpha_{1} & \alpha_{2} & \cdots & \alpha_{K-1} \\
\vdots & \vdots & \ddots & \vdots \\
\alpha_{1}^{K-2} & \alpha_{2}^{K-2} & \cdots & \alpha_{K-1}^{K-2}
\end{array}\right)\left(\begin{array}{c}
\left(\alpha_{1}-\alpha_{K}\right) q_{1} \\
\left(\alpha_{2}-\alpha_{K}\right) q_{2} \\
\vdots \\
\left(\alpha_{K-1}-\alpha_{K}\right) q_{K-1}
\end{array}\right)=\mathbf{0} .
$$

But $\alpha_{i} \neq \alpha_{j}$ for $i \neq j$. Thus, we immediately find that $\left(\alpha_{k}-\alpha_{K}\right) q_{k}=0,1 \leq k \leq K-1$, i.e., $q_{1}=q_{2}=$ $\cdots=q_{K-1}=0$, and that $q_{K}=-\sum_{k=1}^{K-1} q_{k}=0$.

Recall that $\tau^{*} p_{k}=\left\langle q_{k}, \xi_{1}\right\rangle_{\text {., }} w=0,1 \leq k \leq K$. Thus, $p_{k}(\neq 0)$ belongs to $\mathscr{P}\left(L^{*}\right)$, and $L^{*} p_{k}=\alpha_{k} p_{k}$ by (42).

Each $\alpha_{k}$ is found an eigenvalue of $L^{*}$, and $p_{k}(\neq 0)$ must be an eigenfunction. In addition,

$$
\begin{aligned}
& d_{k}=\left\langle p_{k}+X_{1}^{*} q_{k}, \zeta\right\rangle=\left\langle p_{k}, \zeta\right\rangle=0, \quad 1 \leq k \leq K, \\
& e_{k}=\left\langle p_{k}+X_{1}^{*} q_{k}, \eta\right\rangle=\left\langle p_{k}, \eta\right\rangle=0, \quad 1 \leq k \leq K .
\end{aligned}
$$

But, this contradicts our assumption (41). Q.E.D. Proof of Theorem 9. We already know that $\sigma\left(B_{1}^{*}\right) \subset$ $\sigma\left(A^{*}\right)$ by Proposition 8. Let $\hat{\lambda}$ be in $\sigma\left(A^{*}\right) \backslash \sigma\left(B_{1}^{*}\right)$.

In view of $(51)$, the relation: $A^{*}(p q)^{\mathrm{T}}=\hat{\lambda}(p q)^{\mathrm{T}}$, $\left(\begin{array}{ll}p & q\end{array}\right)^{\mathrm{T}} \neq\left(\begin{array}{ll}0 & 0\end{array}\right)^{\mathrm{T}}$ means that

$$
\begin{aligned}
& \mathscr{L}^{*} p=\hat{\lambda} p, \\
& B_{1}^{*} q-\left\langle p+X_{1}^{*} q, \zeta\right\rangle \rho+\left\langle p+X_{1}^{*} q, \eta\right\rangle \tilde{f}=\hat{\lambda} q,
\end{aligned}
$$

where $\tilde{f}=\left(\alpha-B^{*}\right) f$. The calculation of: (the first equation $)+X_{1}^{*} \times($ the second equation) yields that

$$
\begin{aligned}
& \mathscr{L}^{*}\left(p+X_{1}^{*} q\right)-\left\langle p+X_{1}^{*} q, \zeta\right\rangle X_{1}^{*} \rho \\
& +\left\langle p+X_{1}^{*} q, \eta\right\rangle X_{1}^{*} \tilde{f}=\hat{\lambda}\left(p+X_{1}^{*} q\right) .
\end{aligned}
$$

By noting that $\tau^{*}\left(p+X_{1}^{*} q\right)=\tau^{*} p-\left\langle q, \xi_{1}\right\rangle, w^{*}=0$ (see (48)), the function $p+X_{1}^{*} q$ belongs to $\left(L^{*}\right)=$ $\mathscr{Y}\left(L_{g}^{*}\right)$. Thus we see that $L_{g}^{*}\left(p+X_{1}^{*} q\right)=\hat{\lambda}\left(p+X_{1}^{*} q\right)$. Supposing that $\hat{\psi}=p+X_{1}^{*} q=0$, we show a contradiction. In fact, if so, the second equation of (58) becomes $B_{1}^{*} q=\hat{\lambda} q$. Since $\hat{\lambda} \notin \sigma\left(B_{1}^{*}\right)$, however, we see that $q=0$, and $p=-X_{1}^{*} q=0$, or $(p q)^{\mathrm{T}}=\left(\begin{array}{ll}0 & 0\end{array}\right)^{\mathrm{T}}$. Thus, $\hat{\lambda}$ belongs to $\sigma\left(L_{g}^{*}\right)$, and the corresponding eigenvector of $A^{*}$ is given by the form $(p q)^{\mathrm{T}}=\left(\hat{\psi}-X_{1}^{*} q q\right)^{\mathrm{T}}$, where $q \neq 0$. We have also shown that $\sigma\left(A^{*}\right) \subset$ $\sigma\left(L_{g}^{*}\right) \cup \sigma\left(B_{1}^{*}\right)$.

Conversely, let $\{\hat{\lambda}, \hat{\psi}\}$ be an arbitrary eigenpair of $L_{g}^{*}$ such that $\hat{\lambda} \in \sigma\left(L_{g}^{*}\right) \backslash \sigma\left(B_{1}^{*}\right)$. Then we solve the equation: $B_{1}^{*} q-\langle\hat{\psi}, \zeta\rangle \rho+\langle\hat{\psi}, \eta\rangle \hat{f}=\hat{\lambda} q$, the unique solution of which is given by $q=\left(\hat{\lambda}-B_{1}^{*}\right)^{-1}(-\langle\hat{\psi}, \zeta\rangle \rho+\langle\hat{\psi}, \eta\rangle \tilde{f})$. By setting $p=\hat{\psi}-X_{1}^{*} q$, the vector $(p q)^{\mathrm{T}}$ means (46), and clearly satisfies the relation: $A^{*}(p q)^{\mathrm{T}}=\hat{\lambda}(p q)^{\mathrm{T}}$. To show that $q \neq 0$, we suppose the contrary: $q=0$, or $-\langle\hat{\psi}, \zeta\rangle \rho+\langle\hat{\psi}, \eta\rangle \tilde{f}=0$. Then, $p=\hat{\psi} \neq 0$, and $\tau^{*} p=0$. Thus, $\{\hat{\lambda}, p\}$ must be an eigenpair of $L^{*}$. But, this contradicts the assumptions (40) and (41). We have shown that $(p q)^{\mathrm{T}}$ given by (46) is an eigenvector of $A^{*}$. Q.E.D.

Remark. In (45), it is not certain if $\sigma\left(L_{g}^{*}\right) \cap \sigma\left(B_{1}^{*}\right) \neq \varnothing$. This problem seems a pathological one. If $\left(\mu_{i} \omega^{ \pm}-L_{g}^{*}\right) \hat{\psi}=$ $0, \hat{\psi} \neq 0$ for some $i, 1 \leq i \leq n$, and, in addition, $\langle\hat{\psi}, \zeta\rangle \rho_{i}^{ \pm}=\langle\hat{\psi}, \eta\rangle \tilde{f}_{\dot{f}}^{ \pm}$, then the equation $\left(\mu_{i} \omega^{ \pm}-B_{1}^{*}\right) q=$ $-\langle\hat{\psi}, \zeta\rangle \rho+\langle\hat{\psi}, \eta\rangle \tilde{f}$ admits a (non-unique) solution $q$ (see the second equation of (58)). By setting $p=\hat{\psi}-X_{1}^{*} q$, the vector $(p q)^{\mathrm{T}}$ belongs to the eigenspace of $A^{*}$ for $\mu_{i} \omega^{ \pm}$.

\section{REFERENCES}

[1] R. F. Curtain, "Finite Dimensional Compensators for Parabolic Distributed Systems with Unbounded Control and Observation," SIAM Journal on Control and Optimization, Vol. 22, 1984, pp. 255-276. 
http://dx.doi.org/10.1137/0322018

[2] T. Nambu, "On Stabilization of Partial Differential Equations of Parabolic Type: Boundary Observation and Feedback," Funkcialaj Ekvacioj, Vol. 28, No. 3, 1985, pp. 267298.

[3] T. Nambu, "An $L^{2}(\Omega)$-Based Algebraic Approach to Boundary Stabilization for Linear Parabolic Systems," Quarterly of Applied Mathematics, Vol. 62, No. 4, 2004, pp. 711-748.

[4] T. Nambu, "A New Algebraic Approach to Stabilization for Boundary Control Systems of Parabolic Type," Journal of Differential Equations, Vol. 218, No. 1, 2005, pp. 136-158. http://dx.doi.org/10.1016/i.jde.2005.03.013

[5] T. Nambu, "Alternative Algebraic Approach to Stabilization for Linear Parabolic Boundary Control Systems," Mathematics of Control, Signals, and Systems, 2013, in Press. http://dx.doi.org/10.1007/s00498-013-0108-4

[6] Y. Sakawa, "Feedback Stabilization of Linear Diffusion Systems," SIAM Journal on Control and Optimization, Vol. 21, No. 5, 1983, pp. 667-676. http://dx.doi.org/10.1137/0321040

[7] S. Itô, "Diffusion Equations," American Mathematical Society, Providence, 1992.

[8] S. Agmon, "Lectures on Elliptic Boundary Value Problems," Van Nostrand, Princeton, 1965.

[9] A. E. Taylor, "Introduction to Functional Analysis," John Wiley \& Sons, New York, 1958.

[10] T. Nambu, "Stability Enhancement of Output for a Class of Linear Parabolic Systems," Proceedings of Royal Soci- ety Edinburgh, Section A, Vol. 133A, No. 1, 2003, pp. 157-175.

[11] T. Nambu, "Stabilization and Decay of Functionals for Linear Parabolic Control Systems," Proceedings of the Japan Academy, Series A, Vol. 84, No. 2, 2008, pp. 1924. http://dx.doi.org/10.3792/pjaa.84.19

[12] T. Nambu, "On State and Output Stabilization of Linear Parabolic Systems," Funkcialaj Ekvacioj, Vol. 52, No. 3, 2009, pp. 321-341. http://dx.doi.org/10.1619/fesi.52.321

[13] T. Nambu, "Stabilization and a Class of Functionals for Linear Parabolic Control Systems," Proceedings of Royal Society Edinburgh, Section A, Vol. 140, No. 1, 2010, pp. 153-174. http://dx.doi.org/10.1017/S0308210508000978

[14] G. N. Watson, "A Treatise on the Theory of Bessel Functions," Cambridge University Press, Cambridge, 1922.

[15] N. Levinson, "Gap and Density Theorems," American Mathematical Society Colloquium Publications, New York, 1940.

[16] E. C. Titchmarsh, "The Theory of Functions," The Clarendon Press, Oxford, 1939.

[17] Y. Sakawa and T. Matsushita, "Feedback Stabilization of a Class of Distributed Systems and Construction of a State Estimator," IEEE Transactions on Automatic Control, Vol. 20, No. 6, 1975, pp. 748-753. http://dx.doi.org/10.1109/TAC.1975.1101095

[18] R. Courant and D. Hilbert, "Methods of Mathematical Physics, I," Wiley Interscience, New York, 1953. 